\title{
Error analysis of a finite element method for the Willmore flow of graphs
}

\author{
KLAUS DECKELNICK ${ }^{\dagger}$ \\ Institut für Analysis und Numerik, Otto-von-Guericke-Universität Magdeburg, \\ Universitätsplatz 2, 39106 Magdeburg, Germany \\ AND \\ GERHARD DZIUK \\ Institut für Angewandte Mathematik, Universität Freiburg \\ Hermann-Herder-Str. 10, 79104 Freiburg, Germany
}

[Received 21 October 2004 and in revised form 28 September 2005]

\begin{abstract}
The evolution of two-dimensional graphs under Willmore flow is approximated by a continuousin-time finite element method. The highly nonlinear fourth order problem is split into two coupled second order problems using height and a weighted mean curvature as variables. We prove a-priori error estimates for the resulting time-continuous scheme and present results of test calculations.
\end{abstract}

2000 Mathematics Subject Classification: 35K55, 65M15, 65M60.

Keywords: Willmore flow; fourth order parabolic problem; finite element; mixed method; error estimates.

\section{Introduction}

The purpose of this paper is to analyze a finite element scheme for the approximation of twodimensional surfaces in $\mathbb{R}^{3}$ which evolve according to Willmore flow. This flow can be interpreted as the $L^{2}$-gradient flow for the Willmore functional

$$
W(f):=\frac{1}{2} \int_{\Gamma} H^{2} \mathrm{~d} A, \quad \Gamma=f(M),
$$

where $f: M \rightarrow \mathbb{R}^{3}$ is a smooth immersion, $H=\kappa_{1}+\kappa_{2}$ denotes the mean curvature of $\Gamma$ and $\mathrm{d} A$ is the area element. Furthermore, $M$ denotes a fixed two-dimensional surface with or without boundary. Considering normal variations $f_{\epsilon}(x):=f(x)+\epsilon \phi(x) v(x), x \in M$, where $v$ is a unit normal field to $\Gamma$ and $\phi: M \rightarrow \mathbb{R}$ is smooth and vanishes near $\partial M$, one obtains the formula

$$
\left\langle W^{\prime}(f), \phi\right\rangle:=\frac{\mathrm{d}}{\mathrm{d} \epsilon} W\left(f_{\epsilon}\right)_{\mid \epsilon=0}=-\int_{\Gamma} \phi\left(\Delta_{\Gamma} H+\frac{1}{2} H^{3}-2 H K\right) \mathrm{d} A .
$$

Here, $K=\kappa_{1} \kappa_{2}$ is the Gauss curvature of $\Gamma$ and $\Delta_{\Gamma}$ denotes the Laplace-Beltrami operator. The sign of $H$ is chosen in such a way that $H>0$ for a sphere with outward pointing normal.

\footnotetext{
†E-mail: Klaus.Deckelnick@mathematik.uni-magdeburg.de

‡E-mail: gerd.dziuk@mathematik.uni-freiburg.de
} 
For details on this calculation and more information on the Willmore functional see [18]. Given a smooth, oriented surface $\Gamma_{0} \subset \mathbb{R}^{3}$, the Willmore flow problem now consists in finding a family $(\Gamma(t))_{t \in[0, T]}$ of smooth, oriented surfaces which satisfy

$$
\begin{aligned}
V & =\Delta_{\Gamma} H+\frac{1}{2} H^{3}-2 H K \quad \text { on } \Gamma(t), \\
\Gamma(0) & =\Gamma_{0},
\end{aligned}
$$

where $V$ denotes the normal velocity.

In this paper we shall assume that the surfaces $\Gamma(t)$ are graphs over some bounded domain $\Omega \subset \mathbb{R}^{2}$, i.e.

$$
\Gamma(t)=\{(x, u(x, t)) \mid x \in \Omega\}
$$

oriented by the unit normal field

$$
v=\frac{(\nabla u,-1)}{\sqrt{1+|\nabla u|^{2}}},
$$

where $\nabla u=\left(u_{x_{1}}, u_{x_{2}}\right)$. In what follows we shall assume that $\Omega$ has a smooth boundary.

It will be convenient to use the abbreviation

$$
Q:=\sqrt{1+|\nabla u|^{2}}
$$

for the area element. The evolution law 11.1 gives rise to a partial differential equation for the height function $u$. In order to write down this equation we note that the quantities $V, H, K$ and $\Delta_{\Gamma} H$ appearing in 1.1 are expressed in terms of $u$ as follows:

$$
\begin{aligned}
V & =-\frac{u_{t}}{Q}, \quad H=\nabla \cdot\left(\frac{\nabla u}{Q}\right), \quad K=\frac{\operatorname{det} D^{2} u}{Q^{4}}, \\
\Delta_{\Gamma} H & =\frac{1}{Q} \nabla \cdot\left(\left(Q I-\frac{\nabla u \otimes \nabla u}{Q}\right) \nabla H\right),
\end{aligned}
$$

where $D^{2} u$ contains the second space derivatives. The last relation can be rewritten as

$$
\Delta_{\Gamma} H=\nabla \cdot\left(\frac{1}{Q}\left(I-\frac{\nabla u \otimes \nabla u}{Q^{2}}\right) \nabla(Q H)\right)-H \nabla \cdot\left(\frac{1}{Q}\left(I-\frac{\nabla u \otimes \nabla u}{Q^{2}}\right) \nabla Q\right) .
$$

Using the expression for $H$ we conclude that

$$
\frac{1}{Q}\left(I-\frac{\nabla u \otimes \nabla u}{Q^{2}}\right) \nabla Q=\frac{1}{Q}\left(\nabla Q-\frac{\Delta u}{Q} \nabla u\right)+H \frac{\nabla u}{Q}
$$

and a calculation shows that

$$
\nabla \cdot\left(\frac{1}{Q}\left(\nabla Q-\frac{\Delta u}{Q} \nabla u\right)\right)=-2 K .
$$

Inserting (1.8) and 1.9) into 1.7 we obtain

$$
\begin{aligned}
\Delta_{\Gamma} H & =\nabla \cdot\left(\frac{1}{Q}\left(I-\frac{\nabla u \otimes \nabla u}{Q^{2}}\right) \nabla(Q H)\right)+2 H K-H \nabla \cdot\left(H \frac{\nabla u}{Q}\right) \\
& =\nabla \cdot\left(\frac{1}{Q}\left(I-\frac{\nabla u \otimes \nabla u}{Q^{2}}\right) \nabla(Q H)\right)+2 H K-\frac{1}{2} \nabla \cdot\left(\frac{H^{2}}{Q} \nabla u\right)-\frac{1}{2} H^{3} .
\end{aligned}
$$




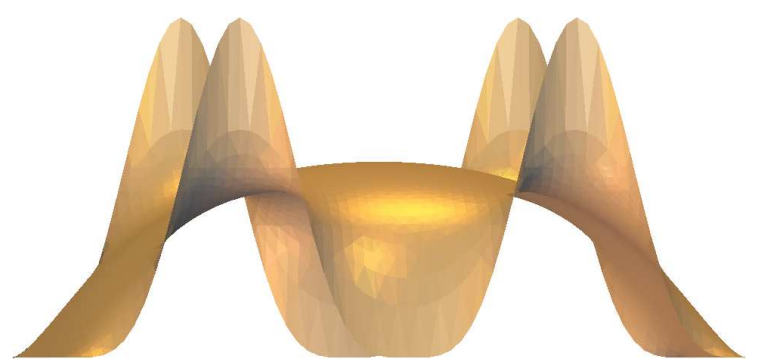

FIG. 1. A stationary solution of $1.10-1.11$ : Willmore surface with prescribed boundary and mean curvature equal to zero on the boundary. The solution was computed with the time dependent algorithm $4.1-4.2$.

Comparing this expression with (1.1) and recalling that $V=-u_{t} / Q$ we obtain the following fourth order parabolic PDE for $u$ :

$$
u_{t}+Q \nabla \cdot\left(\frac{1}{Q}\left(I-\frac{\nabla u \otimes \nabla u}{Q^{2}}\right) \nabla(Q H)\right)-\frac{1}{2} Q \nabla \cdot\left(\frac{H^{2}}{Q} \nabla u\right)=0 \quad \text { in } \Omega \times(0, T) .
$$

Note that the above equation has (after division by $Q$ ) a nice divergence structure in which the Gauss curvature $K$ no longer appears. This structure was exploited by Droske and Rumpf in [8] for a level set approach to Willmore flow. We shall use the boundary conditions

$$
u=g, \quad H=0 \quad \text { on } \partial \Omega \times(0, T),
$$

for a given smooth function $g: \bar{\Omega} \rightarrow \mathbb{R}$. The condition 11.11 is motivated by calculations in [14], where it is shown that if $\Gamma=f(M)$ is stationary for $W$ with respect to variations which keep the boundary of $\Gamma$ fixed, then necessarily $H=0$ on $\partial \Gamma$. Finally, we prescribe the initial condition

$$
u(\cdot, 0)=u_{0} \quad \text { in } \Omega .
$$

In what follows we shall assume that the initial-boundary value problem $1.10-1.12$ has a unique solution $u$, which satisfies

$$
\begin{aligned}
& u \in L^{\infty}\left((0, T) ; H^{4, \infty}(\Omega)\right) \cap L^{2}\left((0, T) ; H^{5}(\Omega)\right), \\
& u_{t} \in L^{\infty}\left((0, T) ; H^{2, \infty}(\Omega)\right) \cap L^{2}\left((0, T) ; H^{3}(\Omega)\right), \\
& u_{t t} \in L^{\infty}\left((0, T) ; L^{\infty}(\Omega)\right) \cap L^{2}\left((0, T) ; H^{1}(\Omega)\right) .
\end{aligned}
$$

Since the focus of our work is on the analysis of a finite element scheme approximating solutions of (1.10)-1.12, we do not address the question of whether a function $u$ satisfying the regularity assumptions (1.13)-(1.15) exists. We expect a positive answer at least for small $T$, but at the moment little seems to be known about boundary value problems for Willmore flow. The situation is different for the evolution of closed surfaces. In [17] it is shown that a unique local solution of 1.1), 1.2 exists provided that $\Gamma_{0}$ is a compact closed immersed and orientable $C^{2, \alpha}$-surface in $\mathbb{R}^{3}$. The solution exists globally in time if $\Gamma_{0}$ is sufficiently close to a sphere in $C^{2, \alpha}$. Using different methods, Kuwert \& Schätzle ([11]) obtain global existence of solutions provided that $\int_{\Gamma_{0}}\left|A^{\circ}\right|^{2}$ is sufficiently small, where $A^{\circ}$ denotes the trace-free part of the second fundamental form. They were subsequently able to remove the smallness assumption and to prove the existence of a global smooth 
solution provided that $W\left(f_{0}\right) \leqslant 16 \pi$, where $\Gamma_{0}=f_{0}\left(S^{2}\right)$ (see [12] and note that our definition differs from the one used in [12] by a factor of 2). The numerical evidence of [13] indicates that the above condition is optimal in the sense that the flow develops a singularity if the initial surface has energy greater than $16 \pi$.

\section{Variational formulation and discretization}

In order to approximate solutions of $1.10-(1.12$ by a finite element scheme, we need to derive a variational formulation of $(1.10)$. To begin, let us introduce

$$
E(p)_{i j}:=\frac{1}{\sqrt{1+|p|^{2}}}\left(\delta_{i j}-\frac{p_{i} p_{j}}{1+|p|^{2}}\right), \quad i, j=1,2, p \in \mathbb{R}^{2} .
$$

It is not difficult to verify that

$$
\begin{aligned}
|E(q)-E(p)| \leqslant c|q-p| & \forall p, q \in \mathbb{R}^{2}, \\
E(p) q \cdot q \geqslant \frac{|q|^{2}}{{\sqrt{1+|p|^{2}}}^{3}} & \forall p, q \in \mathbb{R}^{2} .
\end{aligned}
$$

Now, (1.10) suggests using $w=-Q H$ rather than $H$ as the second variable in a splitting method (cf. [8]). If we divide 1.10 by $Q$, multiply by a test function $\varphi \in H_{0}^{1}(\Omega)$ and integrate by parts we are led to

$$
\begin{aligned}
& \int_{\Omega} \frac{u_{t} \varphi}{Q}+\int_{\Omega} E(\nabla u) \nabla w \cdot \nabla \varphi+\frac{1}{2} \int_{\Omega} \frac{w^{2}}{Q^{3}} \nabla u \cdot \nabla \varphi=0 \quad \forall \varphi \in H_{0}^{1}(\Omega), \\
& \int_{\Omega} \frac{w \zeta}{Q}-\int_{\Omega} \frac{\nabla u \cdot \nabla \zeta}{Q}=0 \quad \forall \zeta \in H_{0}^{1}(\Omega),
\end{aligned}
$$

where the second relation stems from the definition of $w$ and 1.5 .

Before we proceed and base the discretization of our problem on $(2.4),(2.5)$, we want to deduce the decrease in time of the Willmore energy $\frac{1}{2} \int_{\Gamma(t)} H^{2} \mathrm{~d} A=\frac{1}{2} \int_{\Omega} H^{2} Q$ from these relations.

Using $\varphi=u_{t}$ in 2.4 and observing that $\nabla u \cdot \nabla u_{t}=Q_{t} Q$ we deduce

$$
\int_{\Omega} \frac{u_{t}^{2}}{Q}+\int_{\Omega} E(\nabla u) \nabla w \cdot \nabla u_{t}+\frac{1}{2} \int_{\Omega} \frac{w^{2} Q_{t}}{Q^{2}}=0 .
$$

Next, differentiating 2.5 with respect to time gives

$$
\int_{\Omega} \frac{w_{t} \zeta}{Q}-\int_{\Omega} \frac{w \zeta Q_{t}}{Q^{2}}-\int_{\Omega} E(\nabla u) \nabla u_{t} \cdot \nabla \zeta=0 \quad \forall \zeta \in H_{0}^{1}(\Omega) .
$$

If we insert $\zeta=w$ into (2.7) and combine the resulting identity with 2.6 we obtain

$$
0=\int_{\Omega} \frac{u_{t}^{2}}{Q}-\frac{1}{2} \int_{\Omega} \frac{w^{2} Q_{t}}{Q^{2}}+\int_{\Omega} \frac{w w_{t}}{Q}=\int_{\Omega} \frac{u_{t}^{2}}{Q}+\frac{1}{2} \frac{\mathrm{d}}{\mathrm{d} t} \int_{\Omega} \frac{w^{2}}{Q} .
$$

Observing that $\int_{\Omega} w^{2} / Q=\int_{\Omega} H^{2} Q$ we see that the energy decreases in time. 
Let us now turn to the discretization in space. The variational formulation 2.4 - 2.5 suggests using a second order splitting scheme with linear finite elements in order to approximate the pair $(u, w)$. Let $\mathcal{T}_{h}$ be a family of triangulations of $\Omega$ with maximum mesh size $h:=\max _{S \in \mathcal{T}_{h}} \operatorname{diam}(S)$. We suppose that $\bar{\Omega}$ is the union of the elements of $\mathcal{T}_{h}$ so that element edges lying on the boundary are allowed to be curved. Furthermore we suppose that the triangulation is quasiuniform in the sense that there exists a constant $\kappa>0$ (independent of $h$ ) such that each $S \in \mathcal{T}_{h}$ is contained in a ball of radius $\kappa^{-1} h$ and contains a ball of radius $\kappa h$. The discrete space is defined by

$$
X_{h}:=\left\{v_{h} \in C^{0}(\bar{\Omega}) \mid v_{h} \text { is a linear polynomial on each } S \in \mathcal{T}_{h}\right\},
$$

with suitable modifications for boundary elements. There exists an interpolation operator $I_{h}$ : $H^{2}(\Omega) \rightarrow X_{h}$ such that

$$
\left\|v-I_{h} v\right\|+h\left\|\nabla\left(v-I_{h} v\right)\right\| \leqslant c h^{2}\|v\|_{H^{2}(\Omega)} \quad \text { for all } v \in H^{2}(\Omega) .
$$

Furthermore, let $X_{h 0}:=X_{h} \cap H_{0}^{1}(\Omega)$ and suppose that $I_{h} v \in X_{h 0}$ for $v \in H^{2}(\Omega) \cap H_{0}^{1}(\Omega)$. Here and throughout the paper we will denote the $L^{2}(\Omega)$-norm by $\|\cdot\|$.

Our discrete problem now reads: find $\left(u_{h}(t), w_{h}(t)\right), 0 \leqslant t \leqslant T$, such that $u_{h}(t)-I_{h} g \in X_{h 0}$, $w_{h}(t) \in X_{h 0}, u_{h}(0)=u_{0 h} \in X_{h 0}$ and

$$
\begin{aligned}
\int_{\Omega} \frac{u_{h t} \varphi_{h}}{Q_{h}}+\int_{\Omega} E\left(\nabla u_{h}\right) \nabla w_{h} \cdot \nabla \varphi_{h}+\frac{1}{2} \int_{\Omega} \frac{w_{h}^{2}}{Q_{h}^{3}} \nabla u_{h} \cdot \nabla \varphi_{h}=0 & \forall \varphi_{h} \in X_{h 0}, \\
\int_{\Omega} \frac{w_{h} \zeta_{h}}{Q_{h}}-\int_{\Omega} \frac{\nabla u_{h} \cdot \nabla \zeta_{h}}{Q_{h}}=0 & \forall \zeta_{h} \in X_{h 0} .
\end{aligned}
$$

Here, $Q_{h}=\sqrt{1+\left|\nabla u_{h}\right|^{2}}$.

Lemma 2.1 The system (2.10, 2.11) has a unique solution $\left(u_{h}, w_{h}\right)$ on $[0, T]$ for all $T<\infty$.

Proof. Local existence on an interval $\left[0, t_{h}\right)$ follows from the theory of ordinary differential equations. Since $u_{h}(t), w_{h}(t)$ have values in a finite-dimensional space, it is sufficient to bound some norm of $\left(u_{h}, w_{h}\right)$ in order to obtain existence on $[0, T]$. If we repeat the argument which led to 2.8 in the discrete setting, the result is

$$
\int_{0}^{t} \int_{\Omega} \frac{u_{h t}^{2}}{Q_{h}}+\frac{1}{2} \int_{\Omega} \frac{w_{h}^{2}}{Q_{h}} \leqslant C\left(h, u_{0 h}\right), \quad 0 \leqslant t<t_{h} .
$$

Using $\zeta_{h}=u_{h t} \in X_{h 0}$ in 2.11 we obtain (d/d t) $\int_{\Omega} Q_{h}=\int_{\Omega} w_{h} u_{h t} / Q_{h}$, which combined with (2.12) yields

$$
\int_{\Omega} Q_{h}(t) \leqslant c+\left(\int_{0}^{t} \int_{\Omega} \frac{w_{h}^{2}}{Q_{h}}\right)^{1 / 2}\left(\int_{0}^{t} \int_{\Omega} \frac{u_{h t}^{2}}{Q_{h}}\right)^{1 / 2} \leqslant c\left(h, T, u_{0 h}\right), \quad 0 \leqslant t<t_{h} .
$$

This implies that $Q_{h}(x, t) \leqslant c(h, T)$ uniformly in $(x, t) \in \Omega \times\left[0, t_{h}\right)$, and $(2.12)$ then shows that $\left\|u_{h}(t)\right\|,\left\|w_{h}(t)\right\|$ remain bounded on $\left[0, t_{h}\right)$. We leave the proof of uniqueness to the reader.

Our main result is the following error estimate: 
THEOREM 2.2 Assume that (1.10)-(1.12) has a unique solution $u$ on the interval $[0, T]$, which satisfies (1.13)-(1.15). Also suppose that $u_{0 h}=\widehat{u}_{0 h}$, where $\widehat{u}_{0 h}$ is defined as the projection of $u_{0}$ (see 2.17). Then

$$
\begin{aligned}
\sup _{0 \leqslant t \leqslant T}\left\|\left(u-u_{h}\right)(t)\right\|+ & \sup _{0 \leqslant t \leqslant T}\left\|\left(w-w_{h}\right)(t)\right\| \leqslant c h^{2}|\log h|^{2}, \\
& \sup _{0 \leqslant t \leqslant T}\left\|\nabla\left(u-u_{h}\right)(t)\right\| \leqslant c h, \\
& \int_{0}^{T}\left\|u_{t}-u_{h t}\right\|^{2} \mathrm{~d} t \leqslant c h^{4}|\log h|^{4}, \\
& \int_{0}^{T}\left\|\nabla\left(w-w_{h}\right)\right\|^{2} \mathrm{~d} t \leqslant c h^{2} .
\end{aligned}
$$

REMARK 2.3 We expect a similar result if the function $g$ in (1.11) is allowed to depend on time or if we consider a Neumann boundary condition for $u$. Prescribing an inhomogeneous Dirichlet condition for $H$ or replacing it by a Neumann condition is more difficult since our analysis uses the variable $w=-Q H$, so that information on $Q$ on $\partial \Omega \times(0, T)$ is required in order to determine the boundary condition for $w$.

A detailed proof of Theorem 2.2 will be given in Section 3. One of the crucial points of our error analysis is the use of suitable nonlinear Ritz projections of $u$ and $w$ which we introduce next. To begin, let $\widehat{u}_{h}$ be defined by: $\widehat{u}_{h}-I_{h} g \in X_{h 0}$ and

$$
\int_{\Omega} \frac{\nabla \widehat{u}_{h} \cdot \nabla \zeta_{h}}{\widehat{Q}_{h}}=\int_{\Omega} \frac{\nabla u \cdot \nabla \zeta_{h}}{Q} \quad \forall \zeta_{h} \in X_{h 0}
$$

where $\widehat{Q}_{h}=\sqrt{1+\left|\nabla \widehat{u}_{h}\right|^{2}}$. Note that $t$ is just a parameter. Estimates for the error

$$
\rho_{u}:=u-\widehat{u}_{h}
$$

in the time-independent case were first carried out in [10], $L^{\infty}$-estimates are due to [15], [9]. For functions $u$ which depend on time it was proved in [4], [5] that

$$
\begin{gathered}
\sup _{0 \leqslant t \leqslant T}\left\|\rho_{u}(t)\right\|+h \sup _{0 \leqslant t \leqslant T}\left\|\nabla \rho_{u}(t)\right\| \leqslant c h^{2}, \\
\sup _{0 \leqslant t \leqslant T}\left\|\rho_{u}(t)\right\|_{L^{\infty}}+h \sup _{0 \leqslant t \leqslant T}\left\|\nabla \rho_{u}(t)\right\|_{L^{\infty}} \leqslant c h^{2}|\log h|, \\
\sup _{0 \leqslant t \leqslant T}\left\|\rho_{u t}(t)\right\| \leqslant c h^{2}|\log h|^{2}, \\
\sup _{0 \leqslant t \leqslant T}\left\|\nabla \rho_{u t}(t)\right\| \leqslant c h .
\end{gathered}
$$

With the help of $\widehat{u}_{h}$, we next define a projection $\widehat{w}_{h} \in X_{h 0}$ of $w$ as follows:

$$
\int_{\Omega} E\left(\nabla \widehat{u}_{h}\right) \nabla \widehat{w}_{h} \cdot \nabla \varphi_{h}=\int_{\Omega} E(\nabla u) \nabla w \cdot \nabla \varphi_{h}+\frac{1}{2} \int_{\Omega} w^{2}\left(\frac{\nabla u}{Q^{3}}-\frac{\nabla \widehat{u}_{h}}{\widehat{Q}_{h}^{3}}\right) \cdot \nabla \varphi_{h} \quad \forall \varphi_{h} \in X_{h 0} .
$$

The proof of the following bounds on the error

$$
\rho_{w}:=w-\widehat{w}_{h}
$$


will be given in Lemma A.1 of the Appendix:

$$
\begin{aligned}
& \sup _{0 \leqslant t \leqslant T}\left\|\nabla \rho_{w}(t)\right\| \leqslant c h, \\
& \sup _{0 \leqslant t \leqslant T}\left\|\rho_{w}(t)\right\| \leqslant c h^{2}|\log h|, \\
& \sup _{0 \leqslant t \leqslant T}\left\|\nabla \rho_{w t}(t)\right\| \leqslant c h, \\
& \sup _{0 \leqslant t \leqslant T}\left\|\rho_{w t}(t)\right\| \leqslant c h^{2}|\log h|^{2} .
\end{aligned}
$$

Note that (1.13), (1.14), 2.19)-2.21), 2.23)-(2.26) together with interpolation and inverse estimates imply that

$$
\left\|\widehat{u}_{h}\right\|_{W^{1, \infty}},\left\|\widehat{u}_{h t}\right\|_{W^{1, \infty}},\left\|\widehat{w}_{h}\right\|_{W^{1, \infty}},\left\|\widehat{w}_{h t}\right\|_{W^{1, \infty}} \leqslant c
$$

uniformly in $h$.

We end this section with a few remarks on numerical approaches to Willmore flow and related problems. In [13], a finite difference scheme is derived to approximate axisymmetric solutions of evolution laws $V=f\left(\kappa_{1}, \kappa_{2}\right)$. The Willmore flow is studied in detail and numerical evidence is provided that the flow may develop singularities in finite time. [8] derives a level set formulation using the level set function and a weighted mean curvature as variables. The formal similarity to the graph approach motivated our choice of variables. For the parametric approach to Willmore flow, [16] derives a variational form which employs position and mean curvature vector as variables and allows the use of linear finite elements to discretize in space. This approach is subsequently extended in [3] to surfaces with boundaries and applied to problems in surface restoration. We also refer to [2], where numerical simulations of anisotropic surface diffusion are carried out for a surface energy which consists of a strongly anisotropic nonconvex part and the Willmore functional (weighted by a small factor).

Let us finally mention evolution by surface diffusion,

$$
V=\Delta_{\Gamma} H \quad \text { on } \Gamma(t),
$$

which coincides with Willmore flow in the highest order term, but which is simpler in that the nonlinear terms in the principal curvatures are absent. In the graph case, it is possible to write down a splitting method using height and mean curvature as variables. Finite element error bounds both for time-continuous and fully discrete schemes have been derived in [6], [1], [7].

\section{Proof of Theorem 2.2}

Before going into details let us sketch the main ideas in the proof of Theorem 2.2 Combining the variational identities 2.4, 2.5) and 2.10, 2.11) with 2.17) and 2.22 we shall derive corresponding relations for $e_{u}:=\widehat{u}_{h}-u_{h}$ and $e_{w}:=\widehat{w}_{h}-w_{h}$. We then try to mimick the derivation of the a-priori estimate 2.8 in order to gain control on

$$
\int_{0}^{T}\left\|e_{u t}\right\|^{2} \mathrm{~d} t+\sup _{t \in(0, T)}\left\|e_{w}(t)\right\|^{2} .
$$


This program is started in Lemmas 3.4 and 3.5. Unlike the case of 2.6 - 2.8 the integrals involving $Q_{t}$ and the matrix $E$ will no longer cancel and require a subtle analysis. Another difficulty stems from the fact that control of the quantities in (3.1) requires a uniform bound on the discrete area element $Q_{h}$. The proof of such a bound heavily relies on the choice of the nonlinear Ritz projections introduced above and a superconvergence property.

To begin, let $\left(u_{h}, w_{h}\right)$ be the discrete solution and denote by $Q_{h}:=\sqrt{1+\left|\nabla u_{h}\right|^{2}}$ the discrete area element. Furthermore, define

$$
C_{0}:=\sup _{x \in \Omega, 0 \leqslant t \leqslant T} Q(x, t), \quad C_{1}:=\sup _{x \in \Omega, 0 \leqslant t \leqslant T}|w(x, t)| .
$$

The initial condition together with a continuity argument then yields, for small $h$,

$$
\sup _{x \in \Omega} Q_{h}(x, t) \leqslant 2 C_{0}, \quad \sup _{x \in \Omega}\left|w_{h}(x, t)\right| \leqslant 2 C_{1}
$$

for small $t$. Define

$$
T_{h}:=\sup \{t \in[0, T] \mid 3.2 \text { holds on }[0, t]\} .
$$

Our strategy is to first prove the error estimates on the interval $\left[0, T_{h}\right)$ and subsequently use these bounds to show that $T_{h}=T$. Thus, in what follows we shall assume (3.2). Let us decompose the errors $u-u_{h}$ and $w-w_{h}$ according to

$$
\begin{gathered}
u-u_{h}=\rho_{u}+e_{u}, \quad \text { where } \quad e_{u}=\widehat{u}_{h}-u_{h}, \\
w-w_{h}=\rho_{w}+e_{w}, \quad \text { where } \quad e_{w}=\widehat{w}_{h}-w_{h} .
\end{gathered}
$$

Furthermore, it will be convenient to work with the following discrete normals:

$$
\widehat{v}_{h}:=\frac{\left(\nabla \widehat{u}_{h},-1\right)}{\widehat{Q}_{h}}, \quad v_{h}:=\frac{\left(\nabla u_{h},-1\right)}{Q_{h}} .
$$

We infer from Proposition 2 in [5] that

$$
\left|\widehat{v}_{h}-v_{h}\right| \leqslant\left|\nabla\left(\widehat{u}_{h}-u_{h}\right)\right| \leqslant\left(1+\sup _{\Omega}\left|\nabla \widehat{u}_{h}\right|\right) Q_{h}\left|\widehat{v}_{h}-v_{h}\right|,
$$

which, combined with 3.2 and 2.27, yields

$$
\left|\widehat{v}_{h}-v_{h}\right| \leqslant\left|\nabla e_{u}\right| \leqslant(1+c) 2 C_{0}\left|\widehat{v}_{h}-v_{h}\right|
$$

In order to make the error analysis more transparent we split it up into a series of auxiliary results.

LEMMA 3.1 Suppose that $F: \mathbb{R}^{n} \rightarrow \mathbb{R}$ is twice continuously differentiable and that $f \in H_{0}^{1}(\Omega)$. Then

$$
\int_{\Omega}\left(F(\nabla u)-F\left(\nabla \widehat{u}_{h}\right)\right) f=-\int_{\Omega} \nabla \cdot\left(f F^{\prime}(\nabla u)\right) \rho_{u}+R,
$$

where $R$ satisfies

$$
|R| \leqslant C h^{2}|\log h|\|f\|
$$


Proof. Clearly,

$$
\begin{aligned}
\int_{\Omega}\left(F(\nabla u)-F\left(\nabla \widehat{u}_{h}\right)\right) f & =\int_{\Omega} F^{\prime}(\nabla u) \cdot \nabla \rho_{u} f+\int_{0}^{1} \int_{\Omega}\left(F^{\prime}\left(\nabla u-s \nabla \rho_{u}\right)-F^{\prime}(\nabla u)\right) \cdot \nabla \rho_{u} f \\
& =-\int_{\Omega} \nabla \cdot\left(f F^{\prime}(\nabla u)\right) \rho_{u}+R
\end{aligned}
$$

where

$$
|R| \leqslant c\left\|\nabla \rho_{u}\right\|_{L^{\infty}}\left\|\nabla \rho_{u}\right\|\|f\| \leqslant C h^{2}|\log h|\|f\|
$$

by (1.13, 2.18, 2.19, 2.27) and since $F$ is twice continuously differentiable.

Lemma 3.2 For every $\epsilon>0$ there exists $c_{\epsilon}$ such that

$$
\left\|\nabla e_{u}(t)\right\|^{2} \leqslant \epsilon\left\|e_{w}(t)\right\|^{2}+c_{\epsilon}\left\|e_{u}(t)\right\|^{2}+c h^{4}|\log h|^{2}, \quad 0 \leqslant t<T_{h} .
$$

Proof. In view of 2.5, 2.11 and 2.17 we have

$$
\int_{\Omega}\left(\frac{\nabla \widehat{u}_{h}}{\widehat{Q}_{h}}-\frac{\nabla u_{h}}{Q_{h}}\right) \cdot \nabla \varphi_{h}=\int_{\Omega}\left(\frac{w}{Q}-\frac{w_{h}}{Q_{h}}\right) \varphi_{h} \quad \forall \varphi_{h} \in X_{h 0} .
$$

Using $\varphi_{h}=\widehat{u}_{h}-u_{h}=e_{u} \in X_{h 0}$ and applying Lemma 3.1 with $F(p)=1 / \sqrt{1+|p|^{2}}$ and $f=w e_{u}$ we derive

$$
\begin{aligned}
& \int_{\Omega}\left(\frac{\nabla \widehat{u}_{h}}{\widehat{Q}_{h}}-\frac{\nabla u_{h}}{Q_{h}}\right) \cdot \nabla e_{u}=\int_{\Omega}\left(\frac{1}{Q}-\frac{1}{\widehat{Q}_{h}}\right) w e_{u}+\int_{\Omega}\left(\frac{1}{\widehat{Q}_{h}}-\frac{1}{Q_{h}}\right) w e_{u}+\int_{\Omega} \frac{\left(w-w_{h}\right) e_{u}}{Q_{h}} \\
& \quad \leqslant \int_{\Omega} \nabla \cdot\left(w e_{u} \frac{\nabla u}{Q^{3}}\right) \rho_{u}+c h^{2}|\log h|\left\|w e_{u}\right\|+c\left\|\nabla e_{u}\right\|\left\|e_{u}\right\|+\left(\left\|e_{w}\right\|+\left\|\rho_{w}\right\|\right)\left\|e_{u}\right\| \\
& \leqslant c h^{2}\left\|\nabla e_{u}\right\|+c\left(\left\|\nabla e_{u}\right\|+\left\|e_{w}\right\|+h^{2}|\log h|\right)\left\|e_{u}\right\|,
\end{aligned}
$$

in view of 1.13, 2.18) and 2.24). Observing that $\left(\nabla e_{u}, 0\right)^{t}=\widehat{Q}_{h} \widehat{v}_{h}-Q_{h} v_{h}$ we may write

$$
\left(\frac{\nabla \widehat{u}_{h}}{\widehat{Q}_{h}}-\frac{\nabla u_{h}}{Q_{h}}\right) \cdot \nabla e_{u}=\left(\widehat{v}_{h}-v_{h}\right) \cdot\left(\widehat{Q}_{h} \widehat{v}_{h}-Q_{h} v_{h}\right)=\frac{1}{2}\left|\widehat{v}_{h}-v_{h}\right|^{2}\left(\widehat{Q}_{h}+Q_{h}\right) \geqslant c_{0}\left\|\nabla e_{u}\right\|^{2}
$$

by 2.27 and 3.4. Combining this inequality with 3.5 implies the result.

LEMMA 3.3

$$
\left\|\nabla e_{w}(t)\right\|^{2} \leqslant c\left(\left\|\nabla e_{u}(t)\right\|^{2}+\left\|e_{u t}(t)\right\|^{2}+\left\|e_{w}(t)\right\|^{2}+h^{4}|\log h|^{4}\right), \quad 0 \leqslant t<T_{h} .
$$

Proof. First note that in view of the definition of $\widehat{w}_{h}$ we have, for all $\varphi_{h} \in X_{h 0}$,

$$
\begin{aligned}
\int_{\Omega} \frac{\widehat{u}_{h t} \varphi_{h}}{Q_{h}}+\int_{\Omega} E\left(\nabla \widehat{u}_{h}\right) \nabla \widehat{w}_{h} \cdot \nabla \varphi_{h}+\frac{1}{2} \int_{\Omega} \frac{\widehat{w}_{h}^{2}}{\widehat{Q}_{h}^{3}} \nabla \widehat{u}_{h} \cdot \nabla \varphi_{h} \\
\quad=\int_{\Omega} \frac{\left(\widehat{u}_{h t}-u_{t}\right) \varphi_{h}}{Q_{h}}+\int_{\Omega} u_{t}\left(\frac{1}{Q_{h}}-\frac{1}{Q}\right) \varphi_{h}+\frac{1}{2} \int_{\Omega}\left(\widehat{w}_{h}^{2}-w^{2}\right) \frac{\nabla \widehat{u}_{h}}{\widehat{Q}_{h}^{3}} \cdot \nabla \varphi_{h},
\end{aligned}
$$


from which we infer

$$
\begin{array}{r}
\int_{\Omega} \frac{e_{u t} \varphi_{h}}{Q_{h}}+\int_{\Omega}\left(E\left(\nabla \widehat{u}_{h}\right) \nabla \widehat{w}_{h}-E\left(\nabla u_{h}\right) \nabla w_{h}\right) \cdot \nabla \varphi_{h}+\frac{1}{2} \int_{\Omega}\left(\frac{\widehat{w}_{h}^{2}}{\widehat{Q}_{h}^{3}} \nabla \widehat{u}_{h}-\frac{w_{h}^{2}}{Q_{h}^{3}} \nabla u_{h}\right) \cdot \nabla \varphi_{h} \\
=-\int_{\Omega} \frac{\rho_{u t} \varphi_{h}}{Q_{h}}+\int_{\Omega} u_{t}\left(\frac{1}{Q_{h}}-\frac{1}{Q}\right) \varphi_{h}+\frac{1}{2} \int_{\Omega}\left(\widehat{w}_{h}^{2}-w^{2}\right) \frac{\nabla \widehat{u}_{h}}{\widehat{Q}_{h}^{3}} \cdot \nabla \varphi_{h}
\end{array}
$$

Inserting $\varphi_{h}=e_{w}$ into 3.6 we derive

$$
\begin{array}{r}
\int_{\Omega}\left(E\left(\nabla \widehat{u}_{h}\right) \nabla \widehat{w}_{h}-E\left(\nabla u_{h}\right) \nabla w_{h}\right) \cdot \nabla e_{w}=-\int_{\Omega} \frac{e_{u t} e_{w}}{Q_{h}}-\frac{1}{2} \int_{\Omega}\left(\frac{\widehat{w}_{h}^{2}}{\widehat{Q}_{h}^{3}} \nabla \widehat{u}_{h}-\frac{w_{h}^{2}}{Q_{h}^{3}} \nabla u_{h}\right) \cdot \nabla e_{w} \\
-\int_{\Omega} \frac{\rho_{u t} e_{w}}{Q_{h}}+\int_{\Omega} u_{t}\left(\frac{1}{Q_{h}}-\frac{1}{Q}\right) e_{w}+\frac{1}{2} \int_{\Omega}\left(\widehat{w}_{h}^{2}-w^{2}\right) \frac{\nabla \widehat{u}_{h}}{\widehat{Q}_{h}^{3}} \cdot \nabla e_{w} .
\end{array}
$$

Using (2.3), 2.2], 2.27] and the fact that $Q_{h} \leqslant 2 C_{0}$ we obtain

$$
\begin{aligned}
\int_{\Omega}\left(E\left(\nabla \widehat{u}_{h}\right) \nabla \widehat{w}_{h}-E\left(\nabla u_{h}\right) \nabla w_{h}\right) \cdot \nabla e_{w} \\
=\int_{\Omega} E\left(\nabla u_{h}\right) \nabla e_{w} \cdot \nabla e_{w}+\int_{\Omega}\left(E\left(\nabla \widehat{u}_{h}\right)-E\left(\nabla u_{h}\right)\right) \nabla \widehat{w}_{h} \cdot \nabla e_{w} \\
\geqslant \frac{1}{\sqrt{1+4 C_{0}^{2}}}\left\|\nabla e_{w}\right\|^{2}-c\left\|\nabla e_{u}\right\|\left\|\nabla e_{w}\right\| \geqslant \frac{1}{2 \sqrt{1+4 C_{0}^{2}}}\left\|\nabla e_{w}\right\|^{2}-c\left\|\nabla e_{u}\right\|^{2} .
\end{aligned}
$$

Furthermore, we infer from Lemma 3.1 that

$$
\begin{aligned}
\int_{\Omega} u_{t}\left(\frac{1}{Q_{h}}-\frac{1}{Q}\right) e_{w} & =\int_{\Omega}\left(\frac{1}{Q_{h}}-\frac{1}{\widehat{Q}_{h}}\right) u_{t} e_{w}-\int_{\Omega}\left(\frac{1}{Q}-\frac{1}{\widehat{Q}_{h}}\right) u_{t} e_{w} \\
& \leqslant c\left\|\nabla e_{u}\right\|\left\|e_{w}\right\|-\int_{\Omega} \nabla \cdot\left(u_{t} e_{w} \frac{\nabla u}{Q^{3}}\right) \rho_{u}+c h^{2}|\log h|\left\|u_{t} e_{w}\right\| \\
& \leqslant c\left\|\nabla e_{u}\right\|\left\|e_{w}\right\|+c h^{2}\left\|\nabla e_{w}\right\|+c h^{2}|\log h|\left\|e_{w}\right\|,
\end{aligned}
$$

by (2.18). The remaining terms are estimated in a straightforward manner so that we finally obtain

$$
\begin{aligned}
\frac{1}{2{\sqrt{1+4 C_{0}^{2}}}^{3}}\left\|\nabla e_{w}\right\|^{2} \leqslant & c\left\|\nabla e_{u}\right\|^{2}+\left\|e_{u t}\right\|\left\|e_{w}\right\|+c\left(\left\|e_{w}\right\|+\left\|\nabla e_{u}\right\|\right)\left\|\nabla e_{w}\right\|+\left\|\rho_{u t}\right\|\left\|e_{w}\right\| \\
& +c\left\|\nabla e_{u}\right\|\left\|e_{w}\right\|+c h^{2}\left\|\nabla e_{w}\right\|+c h^{2}|\log h|\left\|e_{w}\right\|+c\left\|\rho_{w}\right\|\left\|\nabla e_{w}\right\| .
\end{aligned}
$$

The result now follows from Young's inequality, 2.20) and 2.24].

The following two lemmas constitute the first steps in proving Theorem 2.2. As mentioned earlier, the strategy is to mimick the derivation of 2.8 . 
LEMMA 3.4 For $0 \leqslant t<T_{h}$ we have

$$
\begin{array}{r}
\frac{1}{2 C_{0}}\left\|e_{u t}\right\|^{2}+\int_{\Omega}\left(E\left(\nabla \widehat{u}_{h}\right) \nabla \widehat{w}_{h}-E\left(\nabla u_{h}\right) \nabla w_{h}\right) \cdot \nabla e_{u t}+\frac{1}{2} \int_{\Omega}\left(\frac{\widehat{w}_{h}^{2}}{\widehat{Q}_{h}^{3}} \nabla \widehat{u}_{h}-\frac{w_{h}^{2}}{Q_{h}^{3}} \nabla u_{h}\right) \cdot \nabla e_{u t} \\
\leqslant-\frac{\mathrm{d}}{\mathrm{d} t} \int_{\Omega} u_{t} \frac{\nabla u}{Q^{3}} \cdot \nabla e_{u} \rho_{u}+\frac{1}{2} \frac{\mathrm{d}}{\mathrm{d} t} \int_{\Omega}\left(\widehat{w}_{h}^{2}-w^{2}\right) \frac{\nabla \widehat{u}_{h}}{\widehat{Q}_{h}^{3}} \cdot \nabla e_{u}+c\left\|\nabla e_{u}\right\|^{2}+c h^{4}|\log h|^{4} .
\end{array}
$$

Proof. Inserting $\varphi_{h}=e_{u t}$ into (3.6) and recalling 3.2) we obtain

$$
\begin{aligned}
& \frac{1}{2 C_{0}}\left\|e_{u t}\right\|^{2}+\int_{\Omega}\left(E\left(\nabla \widehat{u}_{h}\right) \nabla \widehat{w}_{h}-E\left(\nabla u_{h}\right) \nabla w_{h}\right) \cdot \nabla e_{u t}+\frac{1}{2} \int_{\Omega}\left(\frac{\widehat{w}_{h}^{2}}{\widehat{Q}_{h}^{3}} \nabla \widehat{u}_{h}-\frac{w_{h}^{2}}{Q_{h}^{3}} \nabla u_{h}\right) \cdot \nabla e_{u t} \\
& \leqslant-\int_{\Omega} \frac{\rho_{u t} e_{u t}}{Q_{h}}+\int_{\Omega} u_{t}\left(\frac{1}{Q_{h}}-\frac{1}{\widehat{Q}_{h}}\right) e_{u t}+\int_{\Omega} u_{t}\left(\frac{1}{\widehat{Q}_{h}}-\frac{1}{Q}\right) e_{u t}+\frac{1}{2} \int_{\Omega}\left(\widehat{w}_{h}^{2}-w^{2}\right) \frac{\nabla \widehat{u}_{h}}{\widehat{Q}_{h}^{3}} \cdot \nabla e_{u t} \\
& \equiv I+I I+I I I+I V .
\end{aligned}
$$

We infer from 2.20) that

$$
|I|+|I I| \leqslant\left\|\rho_{u t}\right\|\left\|e_{u t}\right\|+c\left\|\nabla e_{u}\right\|\left\|e_{u t}\right\| \leqslant \epsilon\left\|e_{u t}\right\|^{2}+c_{\epsilon}\left(h^{4}|\log h|^{4}+\left\|\nabla e_{u}\right\|^{2}\right),
$$

while Lemma 3.1 with $F(p)=1 / \sqrt{1+|p|^{2}}$ and $f=u_{t} e_{u t}$ yields

$$
\begin{aligned}
I I I=-\int_{\Omega} \nabla \cdot\left(u_{t} e_{u t} \frac{\nabla u}{Q^{3}}\right) \rho_{u}+R= & -\int_{\Omega} \nabla \cdot\left(u_{t} \frac{\nabla u}{Q^{3}}\right) e_{u t} \rho_{u}-\frac{\mathrm{d}}{\mathrm{d} t} \int_{\Omega} u_{t} \frac{\nabla u}{Q^{3}} \cdot \nabla e_{u} \rho_{u} \\
& +\int_{\Omega}\left(u_{t} \frac{\nabla u}{Q^{3}}\right)_{t} \cdot \nabla e_{u} \rho_{u}+\int_{\Omega} u_{t} \frac{\nabla u}{Q^{3}} \cdot \nabla e_{u} \rho_{u t}+R,
\end{aligned}
$$

where $|R| \leqslant C h^{2}|\log h|\left\|u_{t} e_{u t}\right\|$. Thus,

$$
\begin{aligned}
I I I & \leqslant-\frac{\mathrm{d}}{\mathrm{d} t} \int_{\Omega} u_{t} \frac{\nabla u}{Q^{3}} \cdot \nabla e_{u} \rho_{u}+c\left\|e_{u t}\right\|\left(\left\|\rho_{u}\right\|+h^{2}|\log h|\right)+c\left\|\nabla e_{u}\right\|\left(\left\|\rho_{u}\right\|+\left\|\rho_{u t}\right\|\right) \\
& \leqslant-\frac{\mathrm{d}}{\mathrm{d} t} \int_{\Omega} u_{t} \frac{\nabla u}{Q^{3}} \cdot \nabla e_{u} \rho_{u}+\epsilon\left\|e_{u t}\right\|^{2}+c\left\|\nabla e_{u}\right\|^{2}+c_{\epsilon} h^{4}|\log h|^{4}
\end{aligned}
$$

by 2.18, 2.20. Finally,

$$
\begin{aligned}
I V= & \frac{1}{2} \frac{\mathrm{d}}{\mathrm{d} t} \int_{\Omega}\left(\widehat{w}_{h}^{2}-w^{2}\right) \frac{\nabla \widehat{u}_{h}}{\widehat{Q}_{h}^{3}} \cdot \nabla e_{u}-\int_{\Omega}\left(\widehat{w}_{h} \widehat{w}_{h t}-w w_{t}\right) \frac{\nabla \widehat{u}_{h}}{\widehat{Q}_{h}^{3}} \cdot \nabla e_{u} \\
& -\frac{1}{2} \int_{\Omega}\left(\widehat{w}_{h}^{2}-w^{2}\right)\left(\frac{\nabla \widehat{u}_{h}}{\widehat{Q}_{h}^{3}}\right)_{t} \cdot \nabla e_{u} .
\end{aligned}
$$

We infer from 2.21, 2.24 and 2.27 that

$$
\begin{aligned}
I V & \leqslant \frac{1}{2} \frac{\mathrm{d}}{\mathrm{d} t} \int_{\Omega}\left(\widehat{w}_{h}^{2}-w^{2}\right) \frac{\nabla \widehat{u}_{h}}{\widehat{Q}_{h}^{3}} \cdot \nabla e_{u}+c\left\|\nabla e_{u}\right\|\left(\left\|\rho_{w}\right\|+\left\|\rho_{w t}\right\|\right) \\
& \leqslant \frac{1}{2} \frac{\mathrm{d}}{\mathrm{d} t} \int_{\Omega}\left(\widehat{w}_{h}^{2}-w^{2}\right) \frac{\nabla \widehat{u}_{h}}{\widehat{Q}_{h}^{3}} \cdot \nabla e_{u}+c\left\|\nabla e_{u}\right\|^{2}+c h^{4}|\log h|^{4}
\end{aligned}
$$

by 2.24, 2.26). Summing 3.7 -3.9 and choosing $\epsilon$ sufficiently small yields the result. 
LEMMA 3.5 For $0 \leqslant t<T_{h}$ we have

$$
\begin{aligned}
\frac{1}{2} \frac{\mathrm{d}}{\mathrm{d} t} \int_{\Omega} \frac{e_{w}^{2}}{Q_{h}}-\frac{1}{2} \int_{\Omega} \frac{e_{w}^{2}}{Q_{h}^{2}} Q_{h t}-\int_{\Omega} \widehat{w}_{h}\left(\frac{\widehat{Q}_{h t}}{\widehat{Q}_{h}^{2}}-\frac{Q_{h t}}{Q_{h}^{2}}\right) e_{w} \\
\quad-\int_{\Omega}\left(E\left(\nabla \widehat{u}_{h}\right) \nabla \widehat{u}_{h t}-E\left(\nabla u_{h}\right) \nabla u_{h t}\right) \cdot \nabla e_{w} \\
\leqslant \epsilon\left\|\nabla e_{w}\right\|^{2}+c_{\epsilon}\left(\left\|\nabla e_{u}\right\|^{2}+\left\|e_{w}\right\|^{2}\right)+c_{\epsilon} h^{4}|\log h|^{4} .
\end{aligned}
$$

Proof. Starting from 2.5) and recalling the definition of $\widehat{u}_{h}$ we infer

$$
\int_{\Omega} \frac{w \xi_{h}}{Q}=\int_{\Omega} \frac{\nabla u \cdot \nabla \xi_{h}}{Q}=\int_{\Omega} \frac{\nabla \widehat{u}_{h} \cdot \nabla \xi_{h}}{\widehat{Q}_{h}} \quad \forall \xi_{h} \in X_{h 0},
$$

from which we obtain after differentiation with respect to time

$$
\int_{\Omega} \frac{w_{t} \xi_{h}}{Q}-\int_{\Omega} \frac{w \xi_{h}}{Q^{2}} Q_{t}-\int_{\Omega} E\left(\nabla \widehat{u}_{h}\right) \nabla \widehat{u}_{h t} \cdot \nabla \xi_{h}=0 \quad \forall \xi_{h} \in X_{h 0} .
$$

Similarly

$$
\int_{\Omega} \frac{w_{h t} \xi_{h}}{Q_{h}}-\int_{\Omega} \frac{w_{h} \xi_{h}}{Q_{h}^{2}} Q_{h t}-\int_{\Omega} E\left(\nabla u_{h}\right) \nabla u_{h t} \cdot \nabla \xi_{h}=0 \quad \forall \xi_{h} \in X_{h 0} .
$$

Taking the difference of the above relations we obtain

$$
\begin{aligned}
\int_{\Omega} \frac{e_{w t} \xi_{h}}{Q_{h}} & -\int_{\Omega} \frac{e_{w} \xi_{h}}{Q_{h}^{2}} Q_{h t}-\int_{\Omega} \widehat{w}_{h}\left(\frac{\widehat{Q}_{h t}}{\widehat{Q}_{h}^{2}}-\frac{Q_{h t}}{Q_{h}^{2}}\right) \xi_{h} \\
& -\int_{\Omega}\left(E\left(\nabla \widehat{u}_{h}\right) \nabla \widehat{u}_{h t}-E\left(\nabla u_{h}\right) \nabla u_{h t}\right) \cdot \nabla \xi_{h} \\
= & \int_{\Omega} \widehat{w}_{h t}\left(\frac{1}{Q_{h}}-\frac{1}{Q}\right) \xi_{h}-\int_{\Omega} \rho_{w t} \frac{1}{Q} \xi_{h}+\int_{\Omega} \rho_{w} \frac{Q_{t}}{Q^{2}} \xi_{h}-\int_{\Omega} \widehat{w}_{h}\left(\frac{\widehat{Q}_{h t}}{\widehat{Q}_{h}^{2}}-\frac{Q_{t}}{Q^{2}}\right) \xi_{h} .
\end{aligned}
$$

If we use $\xi_{h}=e_{w}$ the result is

$$
\begin{aligned}
\frac{1}{2} \frac{\mathrm{d}}{\mathrm{d} t} \int_{\Omega} & \frac{e_{w}^{2}}{Q_{h}}-\frac{1}{2} \int_{\Omega} \frac{e_{w}^{2} Q_{h t}}{Q_{h}^{2}}-\int_{\Omega} \widehat{w}_{h}\left(\frac{\widehat{Q}_{h t}}{\widehat{Q}_{h}^{2}}-\frac{Q_{h t}}{Q_{h}^{2}}\right) e_{w} \\
& -\int_{\Omega}\left(E\left(\nabla \widehat{u}_{h}\right) \nabla \widehat{u}_{h t}-E\left(\nabla u_{h}\right) \nabla u_{h t}\right) \cdot \nabla e_{w} \\
= & \int_{\Omega} \widehat{w}_{h t}\left(\frac{1}{Q_{h}}-\frac{1}{Q}\right) e_{w}-\int_{\Omega} \rho_{w t} \frac{1}{Q} e_{w}+\int_{\Omega} \rho_{w} \frac{Q_{t}}{Q^{2}} e_{w}-\int_{\Omega} \widehat{w}_{h}\left(\frac{\widehat{Q}_{h t}}{\widehat{Q}_{h}^{2}}-\frac{Q_{t}}{Q^{2}}\right) e_{w} \\
\equiv & I+\cdots+I V .
\end{aligned}
$$

In order to deal with the first term we apply again Lemma 3.1 with $F(p):=1 / \sqrt{1+|p|^{2}}$ and $f=\widehat{w}_{h t} e_{w}$ :

$$
\begin{aligned}
I & =\int_{\Omega}\left(\frac{1}{Q_{h}}-\frac{1}{\widehat{Q}_{h}}\right) \widehat{w}_{h t} e_{w}+\int_{\Omega}\left(\frac{1}{\widehat{Q}_{h}}-\frac{1}{Q}\right) \widehat{w}_{h t} e_{w} \\
& =\int_{\Omega}\left(\frac{1}{Q_{h}}-\frac{1}{\widehat{Q}_{h}}\right) \widehat{w}_{h t} e_{w}-\int_{\Omega} \nabla \cdot\left(\widehat{w}_{h t} e_{w} \frac{\nabla u}{Q^{3}}\right) \rho_{u}+R,
\end{aligned}
$$


where $|R| \leqslant c h^{2}|\log h|\left\|\widehat{w}_{h t} e_{w}\right\|$. As a consequence,

$$
\begin{aligned}
|I| & \leqslant c\left\|\widehat{w}_{h t}\right\|_{L^{\infty}}\left(\left\|\nabla e_{u}\right\|\left\|e_{w}\right\|+\left\|\nabla e_{w}\right\|\left\|\rho_{u}\right\|+h^{2}|\log h|\left\|e_{w}\right\|\right)+c\left\|\nabla \widehat{w}_{h t}\right\|_{L^{\infty}}\left\|e_{w}\right\|\left\|\rho_{u}\right\| \\
& \leqslant \epsilon\left\|\nabla e_{w}\right\|^{2}+c_{\epsilon} h^{4}|\log h|^{2}+c\left\|\nabla e_{u}\right\|^{2}+c\left\|e_{w}\right\|^{2}
\end{aligned}
$$

by 2.27]. Next, 2.24) and 2.26) imply

$$
|I I+I I I| \leqslant c\left(\left\|\rho_{w t}\right\|+\left\|\rho_{w}\right\|\right)\left\|e_{w}\right\| \leqslant c\left\|e_{w}\right\|^{2}+c h^{4}|\log h|^{4} .
$$

Abbreviating $G_{i}(p):=p_{i} /{\sqrt{1+|p|^{2}}}^{3}$ and $i=1,2$, we obtain

$$
\begin{aligned}
I V= & \int_{\Omega}\left(\frac{\nabla u \cdot \nabla u_{t}}{Q^{3}}-\frac{\nabla \widehat{u}_{h} \cdot \nabla \widehat{u}_{h t}}{\widehat{Q}_{h}^{3}}\right) \widehat{w}_{h} e_{w} \\
= & \int_{\Omega}\left(G_{i}(\nabla u)-G_{i}\left(\nabla \widehat{u}_{h}\right)\right) u_{t x_{i}} \widehat{w}_{h} e_{w}-\int_{\Omega}\left(G_{i}(\nabla u)-G_{i}\left(\nabla \widehat{u}_{h}\right)\right) \rho_{u t x_{i}} \widehat{w}_{h} e_{w} \\
& +\int_{\Omega} G_{i}(\nabla u) \rho_{u t x_{i}} \widehat{w}_{h} e_{w} \\
= & -\int_{\Omega} \nabla \cdot\left(G_{i}^{\prime}(\nabla u) \widehat{w}_{h} e_{w} u_{t x_{i}}\right) \rho_{u}+R-\int_{\Omega}\left(G_{i}(\nabla u)-G_{i}\left(\nabla \widehat{u}_{h}\right)\right) \rho_{u t x_{i}} \widehat{w}_{h} e_{w} \\
& -\int_{\Omega} \frac{\partial}{\partial x_{i}} G_{i}(\nabla u) \rho_{u t} \widehat{w}_{h} e_{w}-\int_{\Omega} G_{i}(\nabla u) \widehat{w}_{h x_{i}} \rho_{u t} e_{w}-\int_{\Omega} G_{i}(\nabla u) e_{w x_{i}} \widehat{w}_{h} \rho_{u t},
\end{aligned}
$$

where $|R| \leqslant c h^{2}|\log h|\left\|\widehat{w}_{h} e_{w} \nabla u_{t}\right\|$. Using 2.27) we can estimate

$$
\begin{aligned}
|I V| \leqslant & c\left\|\widehat{w}_{h}\right\|_{W^{1, \infty}}\left\|e_{w}\right\|_{H^{1}}\left\|\rho_{u}\right\|+c h^{2}|\log h|\left\|e_{w}\right\|+c\left\|\nabla \rho_{u}\right\|_{L^{\infty}}\left\|\nabla \rho_{u t}\right\|\left\|e_{w}\right\| \\
& +c\left\|\rho_{u t}\right\|\left\|e_{w}\right\|+c\left\|\nabla \widehat{w}_{h}\right\|_{L^{\infty}}\left\|\rho_{u t}\right\|\left\|e_{w}\right\|+c\left\|\nabla e_{w}\right\|\left\|\widehat{w}_{h}\right\|_{L^{\infty}}\left\|\rho_{u t}\right\| \\
\leqslant & \epsilon\left\|\nabla e_{w}\right\|^{2}+c_{\epsilon} h^{4}|\log h|^{4}+c\left\|e_{w}\right\|^{2} .
\end{aligned}
$$

Combining $3.10-3.12$ we finally obtain the result.

We are now in a position to complete the proof of Theorem 2.2 It follows from Lemmas 3.4 and 3.5 that

$$
\begin{aligned}
& \frac{1}{2 C_{0}}\left\|e_{u t}\right\|^{2}+\frac{1}{2} \frac{\mathrm{d}}{\mathrm{d} t} \int_{\Omega} \frac{e_{w}^{2}}{Q_{h}} \\
& \quad+\frac{1}{2} \int_{\Omega}\left(\frac{\widehat{w}_{h}^{2}}{\widehat{Q}_{h}^{3}} \nabla \widehat{u}_{h}-\frac{w_{h}^{2}}{Q_{h}^{3}} \nabla u_{h}\right) \cdot \nabla e_{u t}-\frac{1}{2} \int_{\Omega} \frac{e_{w}^{2}}{Q_{h}^{2}} Q_{h t}-\int_{\Omega} \widehat{w}_{h}\left(\frac{\widehat{Q}_{h t}}{\widehat{Q}_{h}^{2}}-\frac{Q_{h t}}{Q_{h}^{2}}\right) e_{w} \\
& \quad+\int_{\Omega}\left(E\left(\nabla \widehat{u}_{h}\right) \nabla \widehat{w}_{h}-E\left(\nabla u_{h}\right) \nabla w_{h}\right) \cdot \nabla e_{u t}-\int_{\Omega}\left(E\left(\nabla \widehat{u}_{h}\right) \nabla \widehat{u}_{h t}-E\left(\nabla u_{h}\right) \nabla u_{h t}\right) \cdot \nabla e_{w} \\
& \leqslant-\frac{\mathrm{d}}{\mathrm{d} t} \int_{\Omega} u_{t} \frac{\nabla u}{Q^{3}} \cdot \nabla e_{u} \rho_{u}+\frac{1}{2} \frac{\mathrm{d}}{\mathrm{d} t} \int_{\Omega}\left(\widehat{w}_{h}^{2}-w^{2}\right) \frac{\nabla \widehat{u}_{h}}{\widehat{Q}_{h}^{3}} \cdot \nabla e_{u} \\
& \quad+c_{\epsilon} h^{4}|\log h|^{4}+\epsilon\left\|\nabla e_{w}\right\|^{2}+c_{\epsilon}\left(\left\|e_{u}\right\|_{H^{1}}^{2}+\left\|e_{w}\right\|^{2}\right) .
\end{aligned}
$$


The main problem now is to deal with the terms appearing in the second and third line of the above inequality. A short calculation shows that

$$
\begin{aligned}
\frac{1}{2}\left(\frac{\widehat{w}_{h}^{2}}{\widehat{Q}_{h}^{3}} \nabla \widehat{u}_{h}-\frac{w_{h}^{2}}{Q_{h}^{3}} \nabla u_{h}\right) \cdot \nabla e_{u t}-\frac{1}{2} \frac{e_{w}^{2}}{Q_{h}^{2}} Q_{h t}-\widehat{w}_{h}\left(\frac{\widehat{Q}_{h t}}{\widehat{Q}_{h}^{2}}-\frac{Q_{h t}}{Q_{h}^{2}}\right) e_{w} \\
\quad=-\frac{1}{2} \widehat{w}_{h}^{2} \frac{\widehat{Q}_{h t}}{\widehat{Q}_{h}^{2}}-\frac{1}{2} \frac{\widehat{w}_{h}^{2}}{\widehat{Q}_{h}^{3}} \nabla \widehat{u}_{h} \cdot \nabla u_{h t}-\frac{1}{2} \frac{w_{h}^{2}}{Q_{h}^{3}} \nabla u_{h} \cdot \nabla \widehat{u}_{h t}+\frac{1}{2} \widehat{w}_{h}^{2} \frac{Q_{h t}}{Q_{h}^{2}}+\widehat{w}_{h} w_{h} \frac{\widehat{Q}_{h t}}{\widehat{Q}_{h}^{2}} \\
\quad \equiv S_{1}+\cdots+S_{5} .
\end{aligned}
$$

Clearly,

$$
S_{1}=\frac{1}{2} \widehat{w}_{h}^{2} \frac{\partial}{\partial t}\left(\frac{1}{\widehat{Q}_{h}}\right), \quad S_{4}=-\frac{1}{2} \widehat{w}_{h}^{2} \frac{\partial}{\partial t}\left(\frac{1}{Q_{h}}\right) .
$$

Observing that $\left|\widehat{v}_{h}-v_{h}\right|^{2}=2-2 \frac{1+\nabla \widehat{u}_{h} \cdot \nabla u_{h}}{\widehat{Q}_{h} Q_{h}}$ we obtain

$$
\begin{aligned}
S_{2}= & -\frac{1}{2} \widehat{w}_{h}^{2} \frac{\partial}{\partial t}\left(\frac{\nabla \widehat{u}_{h} \cdot \nabla u_{h}}{\widehat{Q}_{h}^{3}}\right)+\frac{1}{2} \widehat{w}_{h}^{2} \frac{\nabla \widehat{u}_{h t} \cdot \nabla u_{h}}{\widehat{Q}_{h}^{3}}-\frac{3}{2} \widehat{w}_{h}^{2} \frac{\nabla \widehat{u}_{h} \cdot \nabla u_{h}}{\widehat{Q}_{h}^{4}} \widehat{Q}_{h t} \\
= & -\frac{1}{2} \widehat{w}_{h}^{2} \frac{\partial}{\partial t}\left(\frac{\nabla \widehat{u}_{h} \cdot \nabla u_{h}}{\widehat{Q}_{h}^{3}}\right)+\frac{1}{2} \widehat{w}_{h}^{2} \frac{\nabla \widehat{u}_{h t} \cdot \nabla u_{h}}{\widehat{Q}_{h}^{3}}+\frac{3}{4} \widehat{w}_{h}^{2} \frac{Q_{h} \widehat{Q}_{h t}}{\widehat{Q}_{h}^{3}}\left|\widehat{v}_{h}-v_{h}\right|^{2} \\
& -\frac{3}{2} \widehat{w}_{h}^{2} \frac{Q_{h} \widehat{Q}_{h t}}{\widehat{Q}_{h}^{3}}-\frac{1}{2} \widehat{w}_{h}^{2} \frac{\partial}{\partial t}\left(\frac{1}{\widehat{Q}_{h}^{3}}\right),
\end{aligned}
$$

while

$$
S_{3}=-\frac{1}{2}\left(\widehat{w}_{h}-w_{h}\right)^{2} \frac{\nabla u_{h} \cdot \nabla \widehat{u}_{h t}}{Q_{h}^{3}}-\widehat{w}_{h} w_{h} \frac{\nabla u_{h} \cdot \nabla \widehat{u}_{h t}}{Q_{h}^{3}}+\frac{1}{2} \widehat{w}_{h}^{2} \frac{\nabla u_{h} \cdot \nabla \widehat{u}_{h t}}{Q_{h}^{3}} .
$$

Summation of $S_{1}, \ldots, S_{5}$ yields

$$
\begin{aligned}
S_{1}+\cdots+S_{5}= & \frac{1}{2} \widehat{w}_{h}^{2} \frac{\partial}{\partial t}\left(\frac{1}{\widehat{Q}_{h}}-\frac{1}{Q_{h}}-\frac{\nabla \widehat{u}_{h} \cdot \nabla u_{h}}{\widehat{Q}_{h}^{3}}-\frac{1}{\widehat{Q}_{h}^{3}}\right) \\
& +\frac{3}{4} \widehat{w}_{h}^{2} \frac{Q_{h} \widehat{Q}_{h t}}{\widehat{Q}_{h}^{3}}\left|\widehat{v}_{h}-v_{h}\right|^{2}-\frac{1}{2} e_{w}^{2} \frac{\nabla u_{h} \cdot \nabla \widehat{u}_{h t}}{Q_{h}^{3}} \\
& +\frac{1}{2} \widehat{w}_{h}^{2} \nabla\left(u_{h}-\widehat{u}_{h}\right) \cdot \nabla \widehat{u}_{h t}\left(\frac{1}{\widehat{Q}_{h}^{3}}-\frac{1}{Q_{h}^{3}}\right)+\widehat{w}_{h}^{2} \frac{\nabla u_{h} \cdot \nabla \widehat{u}_{h t}}{Q_{h}^{3}}+\frac{1}{2} \widehat{w}_{h}^{2} \frac{\nabla \widehat{u}_{h} \cdot \nabla \widehat{u}_{h t}}{\widehat{Q}_{h}^{3}} \\
& -\frac{1}{2} \widehat{w}_{h}^{2} \frac{\nabla \widehat{u}_{h} \cdot \nabla \widehat{u}_{h t}}{Q_{h}^{3}}+w_{h} \widehat{w}_{h}\left(\frac{\widehat{Q}_{h t}}{\widehat{Q}_{h}^{2}}-\frac{\nabla u_{h} \cdot \nabla \widehat{u}_{h t}}{Q_{h}^{3}}\right)-\frac{3}{2} \widehat{w}_{h}^{2} \frac{Q_{h} \widehat{Q}_{h t}}{\widehat{Q}_{h}^{3}} \\
= & \frac{1}{2} \widehat{w}_{h}^{2} \frac{\partial}{\partial t}\left(\frac{2}{\widehat{Q}_{h}}-\frac{1}{Q_{h}}-\frac{\nabla \widehat{u}_{h} \cdot \nabla u_{h}}{\widehat{Q}_{h}^{3}}-\frac{1}{\widehat{Q}_{h}^{3}}\right)+\frac{3}{4} \widehat{w}_{h}^{2} \frac{Q_{h} \widehat{Q}_{h t}}{\widehat{Q}_{h}^{3}}\left|\widehat{v}_{h}-v_{h}\right|^{2} \\
& -\frac{1}{2} e_{w}^{2} \frac{\nabla u_{h} \cdot \nabla \widehat{u}_{h t}}{Q_{h}^{3}}+\frac{1}{2} \widehat{w}_{h}^{2} \nabla\left(u_{h}-\widehat{u}_{h}\right) \cdot \nabla \widehat{u}_{h t}\left(\frac{1}{\widehat{Q}_{h}^{3}}-\frac{1}{Q_{h}^{3}}\right) \\
& +\widehat{w}_{h}\left(w_{h}-\widehat{w}_{h}\right)\left(\frac{\widehat{Q}_{h t}}{\widehat{Q}_{h}^{2}}-\frac{\nabla u_{h} \cdot \nabla \widehat{u}_{h t}}{Q_{h}^{3}}\right)-\frac{1}{2} \frac{\widehat{Q}_{h t}}{\widehat{Q}_{h}^{3}}\left(-4 \widehat{Q}_{h}+3 Q_{h}+\frac{\widehat{Q}_{h}^{4}}{Q_{h}^{3}}\right) .
\end{aligned}
$$


A short calculation shows that

$$
D:=\frac{2}{\widehat{Q}_{h}}-\frac{1}{Q_{h}}-\frac{\nabla \widehat{u}_{h} \cdot \nabla u_{h}}{\widehat{Q}_{h}^{3}}-\frac{1}{\widehat{Q}_{h}^{3}}
$$

can be written as

$$
D=\frac{1}{2} \frac{Q_{h}}{\widehat{Q}_{h}^{2}}\left|\widehat{v}_{h}-v_{h}\right|^{2}-\frac{1}{Q_{h} \widehat{Q}_{h}^{2}}\left(\widehat{Q}_{h}-Q_{h}\right)^{2} .
$$

Integration over $\Omega$ together with 3.4 gives

$$
\begin{aligned}
& \frac{1}{2} \int_{\Omega}\left(\frac{\widehat{w}_{h}^{2}}{\widehat{Q}_{h}^{3}} \nabla \widehat{u}_{h}-\frac{w_{h}^{2}}{Q_{h}^{3}} \nabla u_{h}\right) \cdot \nabla e_{u t}-\frac{1}{2} \int_{\Omega} \frac{e_{w}^{2}}{Q_{h}^{2}} Q_{h t}-\int_{\Omega} \widehat{w}_{h}\left(\frac{\widehat{Q}_{h t}}{\widehat{Q}_{h}^{2}}-\frac{Q_{h t}}{Q_{h}^{2}}\right) e_{w} \\
& \geqslant \frac{1}{2} \frac{\mathrm{d}}{\mathrm{d} t} \int_{\Omega} \widehat{w}_{h}^{2} D-\int_{\Omega} \widehat{w}_{h} \widehat{w}_{h t} D-c\left\|\nabla e_{u}\right\|^{2}-c\left\|e_{w}\right\|^{2} \\
&-c \int_{\Omega}\left|e_{w}\right|\left|\frac{\widehat{Q}_{h t}}{\widehat{Q}_{h}^{2}}-\frac{\nabla u_{h} \cdot \nabla \widehat{u}_{h t}}{Q_{h}^{3}}\right|-c \int_{\Omega}\left|-4 \widehat{Q}_{h}+3 Q_{h}+\frac{\widehat{Q}_{h}^{4}}{Q_{h}^{3}}\right| .
\end{aligned}
$$

Since

$$
\frac{\widehat{Q}_{h t}}{\widehat{Q}_{h}^{2}}-\frac{\nabla \widehat{u}_{h t} \cdot \nabla u_{h}}{Q_{h}^{3}}=\frac{\nabla \widehat{u}_{h t}}{\widehat{Q}_{h}^{2}}\left(\frac{\nabla \widehat{u}_{h}}{\widehat{Q}_{h}}-\frac{\nabla u_{h}}{Q_{h}}\right)+\frac{\nabla u_{h}}{Q_{h}} \cdot \nabla \widehat{u}_{h t} \frac{Q_{h}+\widehat{Q}_{h}}{\widehat{Q}_{h}^{2} Q_{h}} \frac{Q_{h}-\widehat{Q}_{h}}{Q_{h}}
$$

we obtain

It is easily shown that

$$
\left|\frac{\widehat{Q}_{h t}}{\widehat{Q}_{h}^{2}}-\frac{\nabla \widehat{u}_{h t} \cdot \nabla u_{h}}{Q_{h}^{3}}\right| \leqslant c\left|\widehat{v}_{h}-v_{h}\right| \leqslant c\left\|\nabla e_{u}\right\| .
$$

$$
\left|-4 \widehat{Q}_{h}+3 Q_{h}+\frac{\widehat{Q}_{h}^{4}}{Q_{h}^{3}}\right| \leqslant c\left(Q_{h}-\widehat{Q}_{h}\right)^{2} \leqslant c\left|\nabla e_{u}\right|^{2} .
$$

Recalling 3.14 we have in conclusion

$$
\begin{array}{r}
\frac{1}{2} \int_{\Omega}\left(\frac{\widehat{w}_{h}^{2}}{\widehat{Q}_{h}^{3}} \nabla \widehat{u}_{h}-\frac{w_{h}^{2}}{Q_{h}^{3}} \nabla u_{h}\right) \cdot \nabla e_{u t}-\frac{1}{2} \int_{\Omega} \frac{e_{w}^{2}}{Q_{h}^{2}} Q_{h t}-\int_{\Omega} \widehat{w}_{h}\left(\frac{\widehat{Q}_{h t}}{\widehat{Q}_{h}^{2}}-\frac{Q_{h t}}{Q_{h}^{2}}\right) e_{w} \\
\geqslant \frac{1}{2} \frac{\mathrm{d}}{\mathrm{d} t} \int_{\Omega} \widehat{w}_{h}^{2}\left\{\frac{1}{2} \frac{Q_{h}}{\widehat{Q}_{h}^{2}}\left|\widehat{v}_{h}-v_{h}\right|^{2}-\frac{1}{Q_{h} \widehat{Q}_{h}^{2}}\left(\widehat{Q}_{h}-Q_{h}\right)^{2}\right\}-c\left(\left\|e_{w}\right\|^{2}+\left\|\nabla e_{u}\right\|^{2}\right) .
\end{array}
$$

Next,

$$
\begin{aligned}
& \left(E\left(\nabla \widehat{u}_{h}\right) \nabla \widehat{w}_{h}-E\left(\nabla u_{h}\right) \nabla w_{h}\right) \cdot \nabla e_{u t}-\left(E\left(\nabla \widehat{u}_{h}\right) \nabla \widehat{u}_{h t}-E\left(\nabla u_{h}\right) \nabla u_{h t}\right) \cdot \nabla e_{w} \\
& =\left(E\left(\nabla \widehat{u}_{h}\right)-E\left(\nabla u_{h}\right)\right) \nabla\left(\widehat{u}_{h t}-u_{h t}\right) \cdot \nabla \widehat{w}_{h}-\left(E\left(\nabla \widehat{u}_{h}\right)-E\left(\nabla u_{h}\right)\right) \nabla \widehat{u}_{h t} \cdot \nabla e_{w} .
\end{aligned}
$$

In order to deal with the first term we introduce $\widehat{P}_{h}, P_{h} \in \mathbb{R}^{3 \times 3}$ by

$$
\widehat{P}_{h, i j}=\delta_{i j}-\widehat{v}_{h i} \widehat{v}_{h j}, \quad P_{h, i j}=\delta_{i j}-v_{h i} v_{h j}, \quad i, j=1,2,3 .
$$


Since $\left(\nabla\left(\widehat{u}_{h}-u_{h}\right), 0\right)^{t}=\widehat{Q}_{h} \widehat{v}_{h}-Q_{h} v_{h}$ we may calculate

$$
\begin{gathered}
\left(\frac{1}{\widehat{Q}_{h}} \widehat{P}_{h}-\frac{1}{Q_{h}} P_{h}\right)\left(\nabla\left(\widehat{u}_{h t}-u_{h t}\right), 0\right)^{t}=\left(\frac{1}{\widehat{Q}_{h}} \widehat{P}_{h}-\frac{1}{Q_{h}} P_{h}\right)\left(\widehat{Q}_{h t} \widehat{v}_{h}+\widehat{Q}_{h} \widehat{v}_{h t}-Q_{h t} v_{h}-Q_{h} v_{h t}\right) \\
=\widehat{v}_{h t}-\frac{Q_{h t}}{\widehat{Q}_{h}} \widehat{P}_{h} v_{h}-\frac{Q_{h}}{\widehat{Q}_{h}} \widehat{P}_{h} v_{h t}-\frac{\widehat{Q}_{h t}}{Q_{h}} P_{h} \widehat{v}_{h}-\frac{\widehat{Q}_{h}}{Q_{h}} P_{h} \widehat{v}_{h t}+v_{h t} \\
=\frac{\partial}{\partial t}\left\{v_{h}-\widehat{v}_{h}-\frac{Q_{h}}{\widehat{Q}_{h}} \widehat{P}_{h} v_{h}\right\}+S,
\end{gathered}
$$

where

$$
S=-\frac{\widehat{Q}_{h t} Q_{h}}{\widehat{Q}_{h}^{2}} \widehat{P}_{h} v_{h}+\frac{Q_{h}}{\widehat{Q}_{h}} \widehat{P}_{h t} v_{h}+2 \widehat{v}_{h t}-\frac{\widehat{Q}_{h t}}{Q_{h}} P_{h} \widehat{v}_{h}-\frac{\widehat{Q}_{h}}{Q_{h}} P_{h} \widehat{v}_{h t} .
$$

Observe that

$$
\widehat{P}_{h} v_{h}=v_{h}-\left(v_{h} \cdot \widehat{v}_{h}\right) \widehat{v}_{h}=v_{h}-\widehat{v}_{h}+\frac{1}{2}\left|\widehat{v}_{h}-v_{h}\right|^{2} \widehat{v}_{h}
$$

and similarly

$$
P_{h} \widehat{v}_{h}=\widehat{v}_{h}-v_{h}+\frac{1}{2}\left|\widehat{v}_{h}-v_{h}\right|^{2} v_{h}
$$

This gives

$$
\begin{aligned}
S= & -\frac{\widehat{Q}_{h t} Q_{h}}{\widehat{Q}_{h}^{2}}\left(\widehat{v}_{h}-v_{h}+\frac{1}{2}\left|\widehat{v}_{h}-v_{h}\right|^{2} \widehat{v}_{h}\right)+\frac{Q_{h}}{\widehat{Q}_{h}}\left(-\left(v_{h} \cdot \widehat{v}_{h}\right) \widehat{v}_{h t}-\left(\widehat{v}_{h t} \cdot v_{h}\right) \widehat{v}_{h}\right) \\
& +2 \widehat{v}_{h t}-\frac{\widehat{Q}_{h t}}{Q_{h}}\left(v_{h}-\widehat{v}_{h}+\frac{1}{2}\left|\widehat{v}_{h}-v_{h}\right|^{2} v_{h}\right)-\frac{\widehat{Q}_{h}}{Q_{h}}\left(\widehat{v}_{h t}-\left(\widehat{v}_{h t} \cdot v_{h}\right) v_{h}\right) \\
= & -\frac{1}{2} \widehat{Q}_{h t}\left|\widehat{v}_{h}-v_{h}\right|^{2}\left(\frac{Q_{h}}{\widehat{Q}_{h}^{2}} \widehat{v}_{h}+\frac{1}{Q_{h}} v_{h}\right)+\frac{\widehat{Q}_{h t}}{\widehat{Q}_{h}}\left(\frac{\widehat{Q}_{h}}{Q_{h}}-\frac{Q_{h}}{\widehat{Q}_{h}}\right)\left(\widehat{v}_{h}-v_{h}\right) \\
& +\left(2-\frac{Q_{h}}{\widehat{Q}_{h}}\left(\widehat{v}_{h} \cdot v_{h}\right)-\frac{\widehat{Q}_{h}}{Q_{h}}\right) \widehat{v}_{h t}+\left(\widehat{v}_{h t} \cdot\left(v_{h}-\widehat{v}_{h}\right)\right)\left(\frac{\widehat{Q}_{h}}{Q_{h}} v_{h}-\frac{Q_{h}}{\widehat{Q}_{h}} \widehat{v}_{h}\right)
\end{aligned}
$$

since $\widehat{v}_{h} \cdot \widehat{v}_{h t}=0$. We infer from 3.2 and 3.4 that

$$
\left|\frac{\widehat{Q}_{h}}{Q_{h}}-\frac{Q_{h}}{\widehat{Q}_{h}}\right|,\left|\frac{\widehat{Q}_{h}}{Q_{h}} v_{h}-\frac{Q_{h}}{\widehat{Q}_{h}} \widehat{v}_{h}\right| \leqslant c\left|\nabla e_{u}\right|,
$$

as well as

$$
\left|2-\frac{Q_{h}}{\widehat{Q}_{h}}\left(\widehat{v}_{h} \cdot v_{h}\right)-\frac{\widehat{Q}_{h}}{Q_{h}}\right|=\left|\frac{1}{2} \frac{Q_{h}}{\widehat{Q}_{h}}\right| \widehat{v}_{h}-\left.v_{h}\right|^{2}-\left.\frac{\left(\widehat{Q}_{h}-Q_{h}\right)^{2}}{\widehat{Q}_{h} Q_{h}}|\leqslant c| \nabla e_{u}\right|^{2},
$$

which implies

$$
|S| \leqslant c\left|\nabla e_{u}\right|^{2} .
$$

In conclusion

$$
\begin{aligned}
\left(E\left(\nabla \widehat{u}_{h}\right)-E\left(\nabla u_{h}\right)\right) & \nabla\left(\widehat{u}_{h t}-u_{h t}\right) \cdot \nabla \widehat{w}_{h} \\
& =\left(\frac{1}{\widehat{Q}_{h}} \widehat{P}_{h}-\frac{1}{Q_{h}} P_{h}\right)\left(\nabla\left(\widehat{u}_{h t}-u_{h t}\right), 0\right)^{t} \cdot\left(\nabla \widehat{w}_{h}, 0\right)^{t} \\
& =\left\{\frac{\partial}{\partial t}\left(\left(\frac{Q_{h}}{\widehat{Q}_{h}}-1\right)\left(\widehat{v}_{h}-v_{h}\right)-\frac{1}{2} \frac{Q_{h}}{\widehat{Q}_{h}} \widehat{v}_{h}-\left.v_{h}\right|^{2} \widehat{v}_{h}\right)+S\right\} \cdot\left(\nabla \widehat{w}_{h}, 0\right)^{t} .
\end{aligned}
$$


If we integrate this relation over $\Omega$ and recall 3.17 and 2.27 we obtain

$$
\begin{aligned}
& \int_{\Omega}\left(E\left(\nabla \widehat{u}_{h}\right)-E\left(\nabla u_{h}\right)\right) \nabla\left(\widehat{u}_{h t}-u_{h t}\right) \cdot \nabla \widehat{w}_{h} \\
& =\frac{\mathrm{d}}{\mathrm{d} t} \int_{\Omega}\left(\left(\frac{Q_{h}}{\widehat{Q}_{h}}-1\right)\left(\widehat{v}_{h}-v_{h}\right)-\frac{1}{2} \frac{Q_{h}}{\widehat{Q}_{h}} \widehat{v}_{h}-\left.v_{h}\right|^{2} \widehat{v}_{h}\right) \cdot\left(\nabla \widehat{w}_{h}, 0\right)^{t} \\
& \quad-\int_{\Omega}\left(\left(\frac{Q_{h}}{\widehat{Q}_{h}}-1\right)\left(\widehat{v}_{h}-v_{h}\right)-\frac{1}{2} \frac{Q_{h}}{\widehat{Q}_{h}}\left|\widehat{v}_{h}-v_{h}\right|^{2} \widehat{v}_{h}\right) \cdot\left(\nabla \widehat{w}_{h t}, 0\right)^{t}+\int_{\Omega} S \cdot\left(\nabla \widehat{w}_{h}, 0\right)^{t} \\
& \geqslant \\
& \frac{\mathrm{d}}{\mathrm{d} t} \int_{\Omega}\left(\left(\frac{Q_{h}}{\widehat{Q}_{h}}-1\right)\left(\widehat{v}_{h}-v_{h}\right)-\frac{1}{2} \frac{Q_{h}}{\widehat{Q}_{h}}\left|\widehat{v}_{h}-v_{h}\right|^{2} \widehat{v}_{h}\right) \cdot\left(\nabla \widehat{w}_{h}, 0\right)^{t}-c\left\|\nabla e_{u}\right\|^{2} .
\end{aligned}
$$

Recalling the definitions of $\hat{P}_{h}, P_{h}$ and observing (3.2) we may estimate

$$
\begin{aligned}
\left|\int_{\Omega}\left(E\left(\nabla \widehat{u}_{h}\right)-E\left(\nabla u_{h}\right)\right) \nabla \widehat{u}_{h t} \cdot \nabla e_{w}\right| & =\left|\int_{\Omega}\left(\frac{1}{\widehat{Q}_{h}} \hat{P}_{h}-\frac{1}{Q_{h}} P_{h}\right)\left(\nabla \widehat{u}_{h t}, 0\right)^{t} \cdot\left(\nabla e_{w}, 0\right)^{t}\right| \\
& \leqslant c \int_{\Omega}\left|\nabla e_{u}\left\|\nabla e_{w} \mid \leqslant \epsilon\right\| \nabla e_{w}\left\|^{2}+c_{\epsilon}\right\| \nabla e_{u} \|^{2} .\right.
\end{aligned}
$$

If we insert (3.15), 3.18) and 3.19) into 3.13 we finally obtain

$$
\begin{aligned}
\frac{1}{2 C_{0}}\left\|e_{u t}\right\|^{2}+\frac{1}{2} \frac{\mathrm{d}}{\mathrm{d} t} \int_{\Omega} \frac{e_{w}^{2}}{Q_{h}} \leqslant & -\frac{\mathrm{d}}{\mathrm{d} t} \int_{\Omega} u_{t} \frac{\nabla u}{Q^{3}} \nabla e_{u} \rho_{u}+\frac{1}{2} \frac{\mathrm{d}}{\mathrm{d} t} \int_{\Omega}\left(\widehat{w}_{h}^{2}-w^{2}\right) \frac{\nabla \widehat{u}_{h}}{\widehat{Q}_{h}^{3}} \cdot \nabla e_{u} \\
& -\frac{1}{2} \frac{\mathrm{d}}{\mathrm{d} t} \int_{\Omega} \widehat{w}_{h}^{2}\left\{\frac{1}{2} \frac{Q_{h}}{\widehat{Q}_{h}^{2}}\left|\widehat{v}_{h}-v_{h}\right|^{2}-\frac{1}{Q_{h} \widehat{Q}_{h}^{2}}\left(\widehat{Q}_{h}-Q_{h}\right)^{2}\right\} \\
& -\frac{\mathrm{d}}{\mathrm{d} t} \int_{\Omega}\left(\left(\frac{Q_{h}}{\widehat{Q}_{h}}-1\right)\left(\widehat{v}_{h}-v_{h}\right)-\frac{1}{2} \frac{Q_{h}}{\widehat{Q}_{h}}\left|\widehat{v}_{h}-v_{h}\right|^{2} \widehat{v}_{h}\right) \cdot\left(\nabla \widehat{w}_{h}, 0\right)^{t} \\
& +\epsilon\left\|\nabla e_{w}\right\|^{2}+c_{\epsilon}\left(\left\|e_{w}\right\|^{2}+\left\|\nabla e_{u}\right\|^{2}+h^{4}|\log h|^{4}\right) .
\end{aligned}
$$

Integrating with respect to time between 0 and $t\left(0 \leqslant t<T_{h}\right)$ and taking into account Lemmas 3.2 and 3.3 yields

$$
\begin{gathered}
\int_{0}^{t}\left\|e_{u t}\right\|^{2} \mathrm{~d} s+\left\|e_{w}(t)\right\|^{2} \leqslant c\left\|\nabla e_{u}(t)\right\|\left(\left\|\rho_{u}(t)\right\|+\left\|e_{w}(t)\right\|+\left\|\nabla e_{u}(t)\right\|\right) \\
+\epsilon \int_{0}^{t}\left\|\nabla e_{w}\right\|^{2} \mathrm{~d} s+c_{\epsilon} \int_{0}^{t}\left(\left\|e_{w}\right\|^{2}+\left\|\nabla e_{u}\right\|^{2}\right) \mathrm{d} s+c_{\epsilon} h^{4}|\log h|^{4} \\
\leqslant \epsilon\left(\left\|e_{w}(t)\right\|^{2}+\int_{0}^{t}\left\|e_{u t}\right\|^{2} \mathrm{~d} s\right)+c_{\epsilon}\left(\left\|e_{u}(t)\right\|^{2}+h^{4}|\log h|^{4}+\int_{0}^{t}\left(\left\|e_{u}\right\|^{2}+\left\|e_{w}\right\|^{2}\right) \mathrm{d} s\right) .
\end{gathered}
$$

After choosing $\epsilon$ sufficiently small we obtain

$$
\int_{0}^{t}\left\|e_{u t}\right\|^{2} \mathrm{~d} s+\left\|e_{w}(t)\right\|^{2} \leqslant c_{1}\left\|e_{u}(t)\right\|^{2}+c \int_{0}^{t}\left(\left\|e_{u}\right\|^{2}+\left\|e_{w}\right\|^{2}\right) \mathrm{d} s+c h^{4}|\log h|^{4} .
$$

On the other hand, in view of $e_{u}(0)=0$ we have

$$
\left\|e_{u}(t)\right\|^{2} \leqslant 2 \int_{0}^{t}\left\|e_{u}\right\|\left\|e_{u t}\right\| \mathrm{d} s \leqslant \frac{1}{2} \int_{0}^{t}\left\|e_{u t}\right\|^{2} \mathrm{~d} s+c \int_{0}^{t}\left\|e_{u}\right\|^{2} \mathrm{~d} s .
$$


If we use this inequality in $(3.20)$ we finally obtain

$$
\int_{0}^{t}\left\|e_{u t}\right\|^{2} \mathrm{~d} s+\left\|e_{w}(t)\right\|^{2}+\left\|e_{u}(t)\right\|^{2} \leqslant c \int_{0}^{t}\left(\left\|e_{u}\right\|^{2}+\left\|e_{w}\right\|^{2}\right) \mathrm{d} s+c h^{4}|\log h|^{4}, \quad 0 \leqslant t<T_{h},
$$

and Gronwall's inequality yields

$$
\left\|e_{w}(t)\right\|^{2}+\left\|e_{u}(t)\right\|^{2} \leqslant c h^{4}|\log h|^{4}, \quad 0 \leqslant t \leqslant T_{h} .
$$

Lemma 3.2 implies that $\left\|\nabla e_{u}(t)\right\| \leqslant c h^{2}|\log h|^{2}$ for $0 \leqslant t \leqslant T_{h}$, which together with 2.19 and an inverse estimate gives

$$
\begin{aligned}
\left\|Q_{h}(t)\right\|_{L^{\infty}} & \leqslant\|Q(t)\|_{L^{\infty}}+c\left\|\nabla\left(u-\widehat{u}_{h}\right)(t)\right\|_{L^{\infty}}+c\left\|\nabla e_{u}(t)\right\|_{L^{\infty}} \\
& \leqslant C_{0}+c h|\log h|+c h|\log h|^{2} \leqslant \frac{3}{2} C_{0},
\end{aligned}
$$

and similarly $\left\|w_{h}(t)\right\|_{L^{\infty}} \leqslant \frac{3}{2} C_{1}, 0 \leqslant t \leqslant T_{h}$, provided that $h \leqslant h_{0}$. Now we are in a position to prove that $T_{h}=T$. If not, the above argument would imply that $Q_{h}\left(x, T_{h}\right) \leqslant \frac{3}{2} C_{0}$ and $\left|w_{h}\left(x, T_{h}\right)\right| \leqslant \frac{3}{2} C_{1}$ for $x \in \bar{\Omega}$, and we could establish 3.2 on $\left[0, T_{h}+\delta\right]$ for some $\delta>0$, contradicting the definition of $T_{h}$. The estimates (2.13)-2.16) now follow from 3.21, Lemma 3.2, Lemma 3.3 and the interpolation results for $\widehat{u}_{h}, \widehat{w}_{h}$.

\section{Numerical results}

For our numerical tests we have to use a time discretization. We have chosen a semi-implicit discretization with respect to the time variable in the spatially discrete scheme 2.10$], 2.11$. For a generic function $v$ we denote its evaluation at the $m$-th time level $t_{m}=m \tau$ by $v^{m}=v\left(\cdot, t_{m}\right)$. The time discretization is then given by:

$$
\begin{gathered}
\frac{1}{\tau} \int_{\Omega} \frac{\left(u_{h}^{m+1}-u_{h}^{m}\right) \varphi_{h}}{Q_{h}^{m}}+\int_{\Omega} E\left(\nabla u_{h}^{m}\right) \nabla w_{h}^{m+1} \cdot \nabla \varphi_{h} \\
+\frac{1}{2} \int_{\Omega} \frac{\left(w_{h}^{m}\right)^{2}}{\left(Q_{h}^{m}\right)^{3}} \nabla u_{h}^{m+1} \cdot \nabla \varphi_{h}=\int_{\Omega} f^{m} \varphi_{h} \quad \forall \varphi_{h} \in X_{h 0}, \\
\int_{\Omega} \frac{w_{h}^{m+1} \zeta_{h}}{Q_{h}^{m}}-\int_{\Omega} \frac{\nabla u_{h}^{m+1} \cdot \nabla \zeta_{h}}{Q_{h}^{m}}=0 \quad \forall \zeta_{h} \in X_{h 0},
\end{gathered}
$$

for $m=0,1, \ldots, m(T)$ with $\tau m(T)=T$ and $Q_{h}^{m}=\sqrt{1+\left|\nabla u_{h}^{m}\right|^{2}}$. We have introduced an additional given right hand side $f$, which we will need for our numerical tests. Denote by $\left\{\varphi_{j}\right\}_{j=1, \ldots, N}$ the usual nodal basis of $X_{h 0}$. Then (4.1), (4.2) represents a linear system for the coefficients of $u_{h}^{m+1}$ and $w_{h}^{m+1}$ in the expansions

$$
u_{h}^{m}-I_{h} g=\sum_{j=1}^{N} U_{j}^{m} \varphi_{j}, \quad w_{h}^{m}=\sum_{j=1}^{N} W_{j}^{m} \varphi_{j} .
$$

Denote by $U^{m}=\left(U_{1}^{m}, \ldots, U_{N}^{m}\right), W^{m}=\left(W_{1}^{m}, \ldots, W_{N}^{m}\right)$ the coefficient vectors and set

$$
M_{i, j}^{m}=\int_{\Omega} \frac{\varphi_{i} \varphi_{j}}{Q_{h}^{m}}, \quad E_{i, j}^{m}=\int_{\Omega} E\left(\nabla u_{h}^{m}\right) \nabla \varphi_{i} \cdot \nabla \varphi_{j}, \quad A_{i, j}^{m}=\int_{\Omega} \frac{\nabla \varphi_{i} \cdot \nabla \varphi_{j}}{Q_{h}^{m}},
$$


as well as

$$
B_{i, j}^{m}=\frac{1}{2} \int_{\Omega} \frac{\left(w_{h}^{m}\right)^{2}}{\left(Q_{h}^{m}\right)^{3}} \nabla \varphi_{i} \cdot \nabla \varphi_{j}, \quad F_{i}^{m}=\int_{\Omega} f^{m} \varphi_{i}
$$

for $i, j=1, \ldots, N$. With these settings we can write the linear system 4.1, , 4.2, in the form

$$
\begin{aligned}
\frac{1}{\tau} M^{m} U^{m+1}+E^{m} W^{m+1}+B^{m} U^{m+1} & =\frac{1}{\tau} M^{m} U^{m}+F^{m}, \\
M^{m} W^{m+1}-A^{m} U^{m+1} & =0 .
\end{aligned}
$$

Eliminating $W^{m+1}$ from the first equation by inverting the (weighted) mass matrix in the second equation leads to the linear system

$$
\left(\frac{1}{\tau} M^{m}+E^{m}\left(M^{m}\right)^{-1} A^{m}+B^{m}\right) U^{m+1}=\frac{1}{\tau} M^{m} U^{m}+F^{m} .
$$

We solve this nonsymmetric system by the biconjugate gradient method. In the practical computations we use mass lumping, so that $M^{m}$ becomes a diagonal matrix.

We have tested our algorithm with the help of the following problem: let $\Omega=\left\{x \in \mathbb{R}^{2} \mid\right.$ $|x|<1\},(0, T)=(0,0.5)$ and

$$
u(x, t)=2.5 \cos (2 \pi t)(|x|-1)^{3}|x|^{5} .
$$

The function $f$ is calculated in such a way that $u$ is a solution of the PDE

$$
\frac{u_{t}}{Q}+\nabla \cdot\left(\frac{1}{Q}\left(I-\frac{\nabla u \otimes \nabla u}{Q^{2}}\right) \nabla(Q H)\right)-\frac{1}{2} \nabla \cdot\left(\frac{H^{2}}{Q} \nabla u\right)=f \quad \text { in } \Omega \times(0, T) .
$$

In order not to destroy the second order convergence properties in some norms we choose the time step as $\tau=0.1 h^{2}$. This has proved experimentally to be a good choice for our computations for the Willmore flow of graphs. In Tables 1 and 2 we show the absolute errors for $u$ in the norms

$$
\begin{aligned}
& E_{\infty, 2, u}=\max _{m=1, \ldots, m(T)}\left\|u^{m}-u_{h}^{m}\right\|, \quad \quad E_{\infty, 2, \nabla u}=\max _{m=1, \ldots, m(T)}\left\|\nabla u^{m}-\nabla u_{h}^{m}\right\|, \\
& E_{2,2, u_{t}}=\left(\tau \sum_{m=1}^{m(T)}\left\|u_{t}^{m}-\frac{u_{h}^{m}-u_{h}^{m-1}}{\tau}\right\|^{2}\right)^{1 / 2}, \quad E_{\infty, \infty, u}=\max _{m=1, \ldots, m(T)} \sup _{\Omega}\left|u^{m}-u_{h}^{m}\right|,
\end{aligned}
$$

as well as for $w$ in the norms

$$
\begin{gathered}
E_{\infty, 2, w}=\max _{m=1, \ldots, m(T)}\left\|w^{m}-w_{h}^{m}\right\|, \quad E_{2,2, \nabla w}=\left(\tau \sum_{m=1}^{m(T)}\left\|\nabla w^{m}-\nabla w_{h}^{m}\right\|^{2}\right)^{1 / 2}, \\
E_{\infty, \infty, w}=\max _{m=1, \ldots, m(T)} \sup _{\Omega}\left|w^{m}-w_{h}^{m}\right| .
\end{gathered}
$$

The computational results confirm the theoretical results of Theorem 2.2. Between two spatial grid levels with maximal grid size $h_{1}$ and $h_{2}$ and errors $E\left(h_{1}\right), E\left(h_{2}\right)$ we computed the experimental order of convergence according to

$$
\operatorname{eoc}\left(h_{1}, h_{2}\right)=\log \frac{E\left(h_{1}\right)}{E\left(h_{2}\right)}\left(\log \frac{h_{1}}{h_{2}}\right)^{-1}
$$


TABLE 1

u-errors for the test problem, $\tau=0.1 h^{2}$

\begin{tabular}{|l|ll|ll|ll|ll|}
\hline$h$ & $E_{\infty, 2, u}$ & eoc & $E_{\infty, 2, \nabla u}$ & eoc & $E_{2,2, u_{t}}$ & eoc & $E_{\infty, \infty, u}$ & eoc \\
\hline 1.0 & 0.59486 & - & 1.41194 & - & 1.90956 & - & 1.09159 & - \\
0.73681 & 0.30771 & 2.158 & 0.90635 & 1.451 & 0.97860 & 2.188 & 0.30057 & 4.222 \\
0.42033 & 0.11159 & 1.807 & 0.53259 & 0.947 & 0.50503 & 1.178 & 0.11449 & 1.719 \\
0.22192 & 0.047902 & 1.324 & 0.34495 & 0.680 & 0.23983 & 1.165 & 0.046528 & 1.409 \\
0.11373 & 0.013300 & 1.916 & 0.18205 & 0.956 & 0.070064 & 1.840 & 0.012886 & 1.920 \\
0.057535 & 0.0034522 & 1.979 & 0.094278 & 0.965 & 0.018898 & 1.922 & 0.0033490 & 1.977 \\
\hline
\end{tabular}

TABLE 2

$w$-errors for the test problem, $\tau=0.1 h^{2}$

\begin{tabular}{|l|ll|ll|ll|}
\hline$h$ & $E_{\infty, 2, w}$ & eoc & $E_{2,2, \nabla w}$ & eoc & $E_{\infty, \infty, w}$ & eoc \\
\hline 1.0 & 1.58839 & - & 4.58182 & - & 3.83664 & - \\
0.73681 & 1.44454 & 0.310 & 2.59686 & 1.859 & 1.79998 & 2.477 \\
0.42033 & 0.74736 & 1.174 & 2.99178 & -0.252 & 0.86798 & 1.299 \\
0.22192 & 0.40324 & 0.965 & 1.76851 & 0.823 & 0.41747 & 1.146 \\
0.11373 & 0.11953 & 1.818 & 0.90402 & 1.003 & 0.11833 & 1.885 \\
0.057535 & 0.031087 & 1.976 & 0.44252 & 1.048 & 0.028734 & 2.077 \\
\hline
\end{tabular}
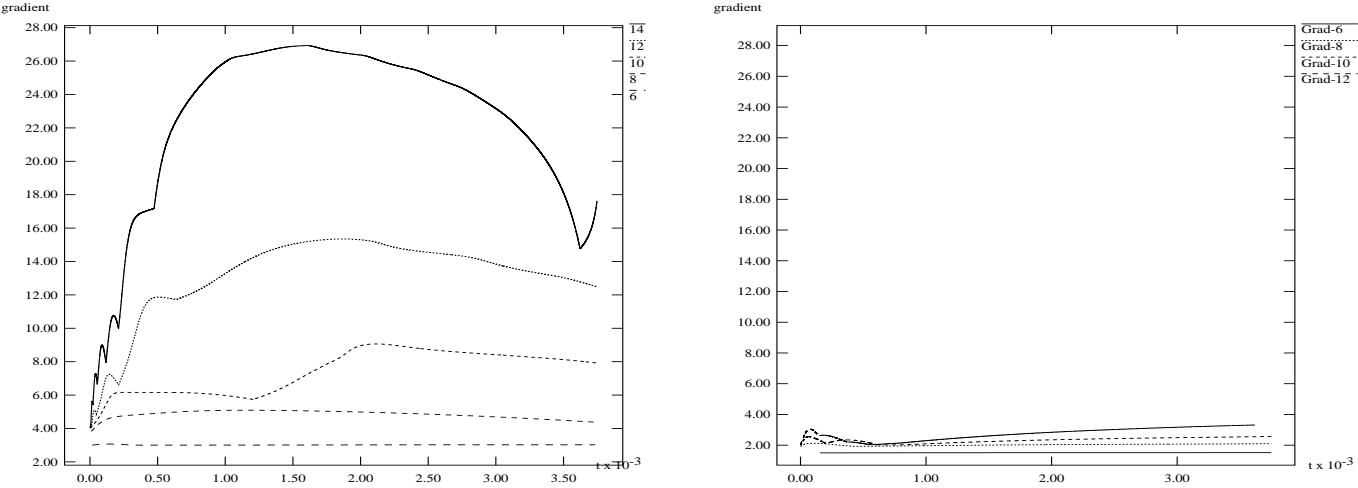

FIG. 2. Maximal gradient of the solution $u$ for initial data (4.6) versus time $t \in(0.0,0.00375)$ for global refinement levels $3,4,5,6$ (left and right), and 7 (left only) of the grid. The plot on the left corresponds to the choice $\delta=1$ in 4.6 , the plot on the right to $\delta=0.5$.

We demonstrate the possible effect that the gradient of the continuous solution may blow up at some time. Figure 2 shows the norm gradient $(t)=\|\nabla u(\cdot, t)\|_{L^{\infty}(\Omega)}$. From Lemma2.1 we know that the discrete solution exists for all times. The continuous one may exist for finite time only. A blow up of the continuous gradient may be deduced practically from the behaviour of the discrete gradient. As initial function for the computations in Figure 2 we have chosen

$$
u_{0}\left(x_{1}, x_{2}\right)=\delta \sin ^{2}\left(\pi\left(1+x_{1}\right)\right) \sin ^{2}\left(\pi x_{1}\right)
$$


on the domain $\Omega=(-1,1) \times(-1,1)$. Figure 2 shows the maximal gradient of the solution corresponding to $\delta=1$ and $\delta=0.5$ respectively on various refinement levels. The results indicate a gradient blow up in the first case while the gradient remains bounded in the latter.

We finally give a computational example for the Willmore flow of a graph. The domain is $\Omega=$ $(-2,2) \times(-2,2)$ and the initial value for $u$ is given by

$$
u_{0}\left(x_{1}, x_{2}\right)=0.75 \sin ^{2}\left(\pi\left(1+x_{1}\right)\right) \sin ^{2}\left(\pi x_{1}\right)+0.1 \sin \left(4 \pi x_{1}\right) \sin \left(5 \pi x_{2}\right) .
$$
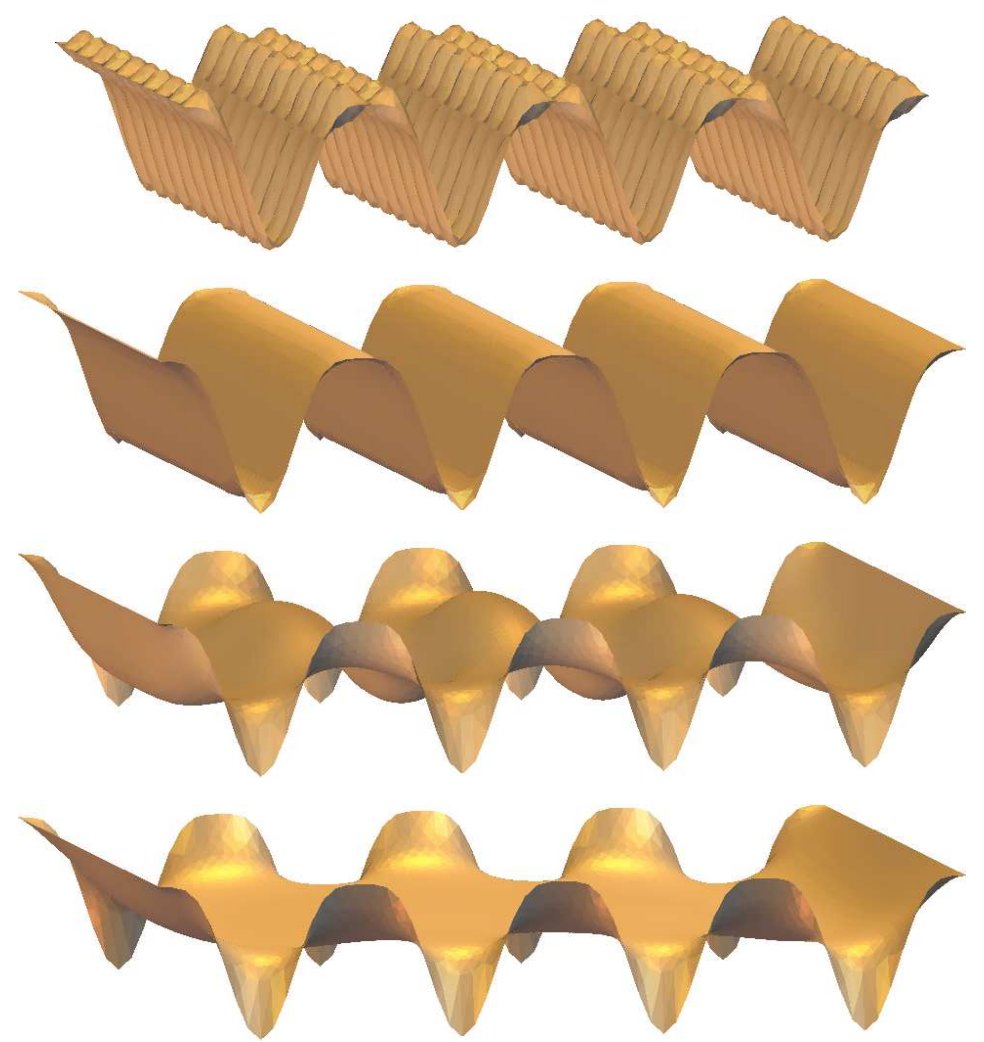

FIG. 3. Graph of $u$ for the time steps $t=0.0,0.000671,0.02448$ and 0.05072 .
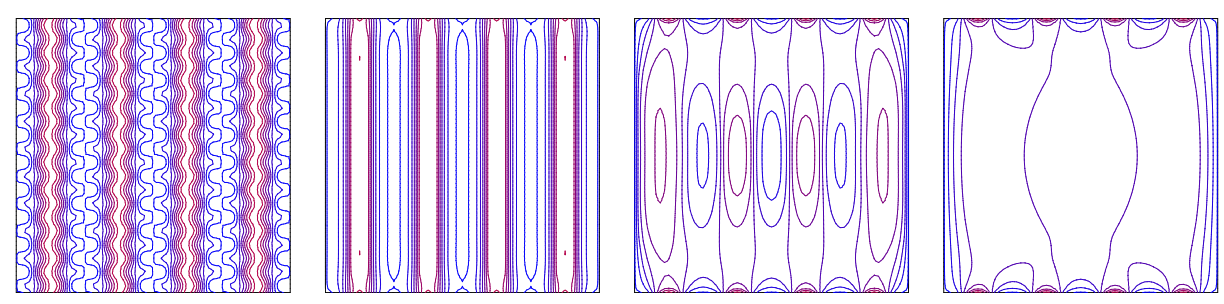

FIG. 4. Level lines of $u$ for the time steps from Figure 3 

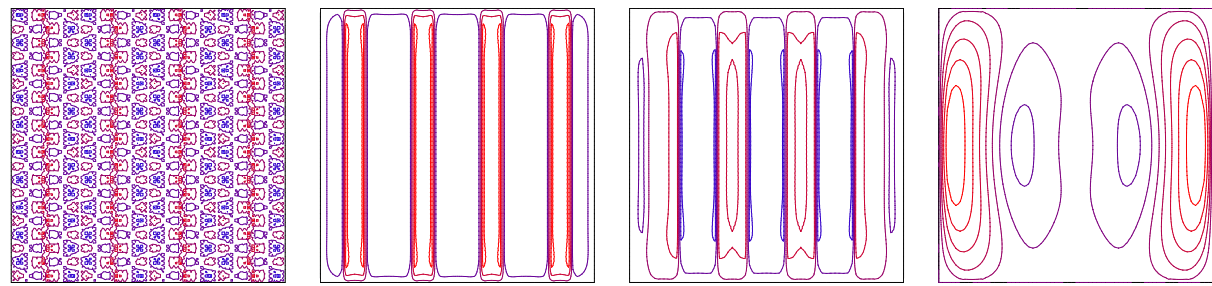

FIG. 5. Level lines of $w$ for the times $t=0.0,0.000671,0.02448,0.05072$.
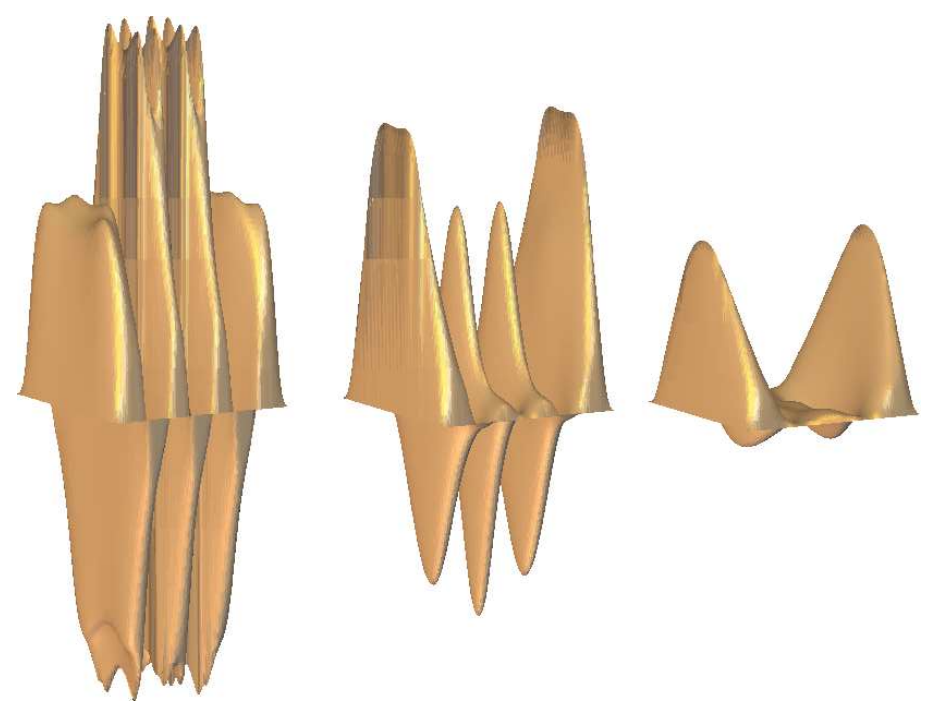

FIG. 6. Graph of $w$ for the time steps $t=0.01837,0.03241$ and $t=0.04279$.

In Figure 3 we show the graph of the solution $u$ at several time steps. Level lines of $u$ are plotted in Figure 4. The levels run from 0.0 to 1.0 with an increment of 0.1 . We show the graph of $w$ for some time steps in Figure 6 and level lines in Figure 5 . The spatial grid size was $h=0.08839$ and in order to capture the rapid smoothing of the solution the time step was chosen as $\tau=6.1035 e-6$.

\section{Appendix}

Lemma A.1 Let $\widehat{w}_{h} \in X_{h 0}$ be given by $(2.22)$. Then

$$
\begin{aligned}
& \sup _{0 \leqslant t \leqslant T}\left\|\nabla\left(w-\widehat{w}_{h}\right)(t)\right\| \leqslant c h \\
& \sup _{0 \leqslant t \leqslant T}\left\|\left(w-\widehat{w}_{h}\right)(t)\right\| \leqslant c h^{2}|\log h|, \\
& \sup _{0 \leqslant t \leqslant T}\left\|\nabla\left(w_{t}-\widehat{w}_{h t}\right)(t)\right\| \leqslant c h, \\
& \sup _{0 \leqslant t \leqslant T}\left\|\left(w_{t}-\widehat{w}_{h t}\right)(t)\right\| \leqslant c h^{2}|\log h|^{2} .
\end{aligned}
$$


Proof. Clearly, 2.22 implies

$$
\begin{aligned}
\int_{\Omega} E\left(\nabla \widehat{u}_{h}\right) \nabla(w & \left.-\widehat{w}_{h}\right) \cdot \nabla \varphi_{h} \\
& =\int_{\Omega}\left(E\left(\nabla \widehat{u}_{h}\right)-E(\nabla u)\right) \nabla w \cdot \nabla \varphi_{h}-\frac{1}{2} \int_{\Omega} w^{2}\left(\frac{\nabla u}{Q^{3}}-\frac{\nabla \widehat{u}_{h}}{\widehat{Q}_{h}^{3}}\right) \cdot \nabla \varphi_{h}
\end{aligned}
$$

for all $\varphi_{h} \in X_{h 0}$. Inserting $\varphi_{h}=I_{h} w-\widehat{w}_{h}$ we obtain

$$
\begin{aligned}
& \int_{\Omega} E\left(\nabla \widehat{u}_{h}\right) \nabla\left(w-\widehat{w}_{h}\right) \cdot \nabla\left(w-\widehat{w}_{h}\right)=\int_{\Omega} E\left(\nabla \widehat{u}_{h}\right) \nabla\left(w-\widehat{w}_{h}\right) \cdot \nabla\left(w-I_{h} w\right) \\
& \quad+\int_{\Omega}\left(E\left(\nabla \widehat{u}_{h}\right)-E(\nabla u)\right) \nabla w \cdot \nabla\left(I_{h} w-\widehat{w}_{h}\right)-\frac{1}{2} \int_{\Omega} w^{2}\left(\frac{\nabla u}{Q^{3}}-\frac{\nabla \widehat{u}_{h}}{\widehat{Q}_{h}^{3}}\right) \cdot \nabla\left(I_{h} w-\widehat{w}_{h}\right),
\end{aligned}
$$

from which we deduce A.1 $)$ in view of $2.3,2.2),(2.18)$, standard interpolation estimates and the uniform boundedness of $Q_{h}$. The $L^{2}$-norm of $w-\widehat{w}_{h}$ is estimated with the help of the usual duality argument. Solve

$$
\begin{aligned}
-\operatorname{div}(E(\nabla u) \nabla z) & =w-\widehat{w}_{h} & & \text { in } \Omega, \\
z & =0 & & \text { on } \partial \Omega .
\end{aligned}
$$

The ellipticity of $E$ together with our smoothness assumptions on $\Omega$ and $u$ implies that the above boundary value problem has a unique solution $z \in H^{2}(\Omega)$ ( $t$ is a parameter) with $\|z\|_{H^{2}} \leqslant$ $c\left\|w-\widehat{w}_{h}\right\|$. Using A.5 with $\varphi_{h}=I_{h} z$ we derive

$$
\begin{aligned}
\left\|w-\widehat{w}_{h}\right\|^{2}= & \int_{\Omega}\left(-\operatorname{div}(E(\nabla u) \nabla z)\left(w-\widehat{w}_{h}\right)\right)=\int_{\Omega} E(\nabla u) \nabla z \cdot \nabla\left(w-\widehat{w}_{h}\right) \\
= & \int_{\Omega}\left(E(\nabla u)-E\left(\nabla \widehat{u}_{h}\right)\right) \nabla z \cdot \nabla\left(w-\widehat{w}_{h}\right)+\int_{\Omega} E\left(\nabla \widehat{u}_{h}\right) \nabla\left(z-I_{h} z\right) \cdot \nabla\left(w-\widehat{w}_{h}\right) \\
& +\int_{\Omega}\left(E\left(\nabla \widehat{u}_{h}\right)-E(\nabla u)\right) \nabla w \cdot \nabla I_{h} z-\frac{1}{2} \int_{\Omega} w^{2}\left(\frac{\nabla u}{Q^{3}}-\frac{\nabla \widehat{u}_{h}}{\widehat{Q}_{h}^{3}}\right) \cdot \nabla I_{h} z \\
\equiv & I+\cdots+I V .
\end{aligned}
$$

We infer from 2.2, 2.19, A.1 and the a-priori estimate for $z$ that

$|I| \leqslant c\left\|\nabla\left(u-\widehat{u}_{h}\right)\right\|_{L^{\infty}}\|\nabla z\|\left\|\nabla\left(w-\widehat{w}_{h}\right)\right\| \leqslant c h^{2}|\log h|\left\|w-\widehat{w}_{h}\right\| \leqslant \epsilon\left\|w-\widehat{w}_{h}\right\|^{2}+c_{\epsilon} h^{4}|\log h|^{2}$.

An interpolation estimate together with A.1 implies

$$
|I I| \leqslant c h\left\|D^{2} z\right\|\left\|\nabla\left(w-\widehat{w}_{h}\right)\right\| \leqslant \epsilon\left\|w-\widehat{w}_{h}\right\|^{2}+c_{\epsilon} h^{4} .
$$

Next, Lemma 3.1 yields

$$
\begin{aligned}
I I I & =\int_{\Omega}\left(E_{i j}\left(\nabla \widehat{u}_{h}\right)-E_{i j}(\nabla u)\right) w_{x_{i}} z_{x_{j}}+\int_{\Omega}\left(E\left(\nabla \widehat{u}_{h}\right)-E(\nabla u)\right) \nabla w \cdot \nabla\left(I_{h} z-z\right) \\
& =\int_{\Omega} \nabla \cdot\left(E_{i j}^{\prime}(\nabla u) w_{x_{i}} z_{x_{j}}\right) \rho_{u}+R+\int_{\Omega}\left(E\left(\nabla \widehat{u}_{h}\right)-E(\nabla u)\right) \nabla w \cdot \nabla\left(I_{h} z-z\right),
\end{aligned}
$$

where $|R| \leqslant c h^{2}|\log h|\|\nabla w\|_{L^{\infty}}\|\nabla z\| \leqslant c h^{2}|\log h|\|\nabla z\|$. This implies

$$
|I I I| \leqslant c\left(\left\|\rho_{u}\right\|+h^{2}|\log h|+h\left\|\nabla \rho_{u}\right\|\right)\|\nabla z\|_{H^{1}} \leqslant \epsilon\left\|w-\widehat{w}_{h}\right\|^{2}+c_{\epsilon} h^{4}|\log h|^{2}
$$


and in a similar way it follows that

$$
|I V| \leqslant \epsilon\left\|w-\widehat{w}_{h}\right\|^{2}+c_{\epsilon} h^{4}|\log h|^{2} .
$$

Combining the estimates for $I, \ldots, I V$ implies (A.2). Let us next turn to the estimates for $w_{t}-\widehat{w}_{h t}$. Differentiating A.5 with respect to time yields

$$
\begin{aligned}
\int_{\Omega} & E\left(\nabla \widehat{u}_{h}\right) \nabla\left(w_{t}-\widehat{w}_{h t}\right) \cdot \nabla \varphi_{h}=-\int_{\Omega} E_{p_{i}}\left(\nabla \widehat{u}_{h}\right) \widehat{u}_{h t, x_{i}} \nabla\left(w-\widehat{w}_{h}\right) \cdot \nabla \varphi_{h} \\
& +\int_{\Omega}\left(E_{p_{i}}\left(\nabla \widehat{u}_{h}\right) \widehat{u}_{h t x_{i}}-E_{p_{i}}(\nabla u) u_{t x_{i}}\right) \nabla w \cdot \nabla \varphi_{h}+\int_{\Omega}\left(E\left(\nabla \widehat{u}_{h}\right)-E(\nabla u)\right) \nabla w_{t} \cdot \nabla \varphi_{h} \\
& -\int_{\Omega} w w_{t}\left(\frac{\nabla u}{Q^{3}}-\frac{\nabla \widehat{u}_{h}}{\widehat{Q}_{h}^{3}}\right) \cdot \nabla \varphi_{h}-\frac{1}{2} \int_{\Omega} w^{2}\left(\frac{\nabla u}{Q^{3}}-\frac{\nabla \widehat{u}_{h}}{\widehat{Q}_{h}^{3}}\right)_{t} \cdot \nabla \varphi_{h}
\end{aligned}
$$

for all $\varphi_{h} \in X_{h 0}$. From this relation for $\varphi_{h}=I_{h} w_{t}-\widehat{w}_{h t}$ it is not difficult to deduce A.3, using (2.2), (2.18), 2.21), (A.1) and an interpolation estimate. It remains to bound $\left\|w_{t}-\widehat{w}_{h t}\right\|$. Denoting by $z$ the solution of

$$
\begin{aligned}
-\operatorname{div}(E(\nabla u) \nabla z) & =w_{t}-\widehat{w}_{h t} & & \text { in } \Omega, \\
z & =0 & & \text { on } \partial \Omega,
\end{aligned}
$$

and proceeding in the same way as above we obtain

$$
\begin{aligned}
\| w_{t}- & \widehat{w}_{h t} \|^{2} \\
= & \int_{\Omega}\left(E(\nabla u)-E\left(\nabla \widehat{u}_{h}\right)\right) \nabla z \cdot \nabla\left(w_{t}-\widehat{w}_{h t}\right)+\int_{\Omega} E\left(\nabla \widehat{u}_{h}\right) \nabla\left(z-I_{h} z\right) \cdot \nabla\left(w_{t}-\widehat{w}_{h t}\right) \\
& -\int_{\Omega} E_{p_{i}}\left(\nabla \widehat{u}_{h}\right) \widehat{u}_{h t x_{i}} \nabla\left(w-\widehat{w}_{h}\right) \cdot \nabla I_{h} z+\int_{\Omega}\left(E_{p_{i}}\left(\nabla \widehat{u}_{h}\right) \widehat{u}_{h t x_{i}}-E_{p_{i}}(\nabla u) u_{t x_{i}}\right) \nabla w \cdot \nabla I_{h} z \\
& +\int_{\Omega}\left(E\left(\nabla \widehat{u}_{h}\right)-E(\nabla u)\right) \nabla w_{t} \cdot \nabla I_{h} z-\int_{\Omega} w w_{t}\left(\frac{\nabla u}{Q^{3}}-\frac{\nabla \widehat{u}_{h}}{\widehat{Q}_{h}^{3}}\right) \cdot \nabla I_{h} z \\
& -\frac{1}{2} \int_{\Omega} w^{2}\left(\frac{\nabla u}{Q^{3}}-\frac{\nabla \widehat{u}_{h}}{\widehat{Q}_{h}^{3}}\right)_{t} \cdot \nabla I_{h} z \\
\equiv & I_{1}+\cdots+I_{7} .
\end{aligned}
$$

Clearly,

$\left|I_{1}\right|+\left|I_{2}\right| \leqslant c\left(\left\|\nabla\left(u-\widehat{u}_{h}\right)\right\|_{L^{\infty}}\|\nabla z\|+c h\|z\|_{H^{2}}\right)\left\|\nabla\left(w_{t}-\widehat{w}_{h t}\right)\right\| \leqslant \epsilon\left\|w_{t}-\widehat{w}_{h t}\right\|^{2}+c_{\epsilon} h^{4}|\log h|^{2}$.

Next,

$$
\begin{aligned}
I_{3}= & \int_{\Omega}\left(E_{p_{i}}(\nabla u) u_{t x_{i}}-E_{p_{i}}\left(\nabla \widehat{u}_{h}\right) \widehat{u}_{h t x_{i}}\right) \nabla\left(w-\widehat{w}_{h}\right) \cdot \nabla z \\
& +\int_{\Omega} E_{p_{i}}\left(\nabla \widehat{u}_{h}\right) \widehat{u}_{h t x_{i}} \nabla\left(w-\widehat{w}_{h}\right) \cdot \nabla\left(z-I_{h} z\right)-\int_{\Omega} E_{p_{i}}(\nabla u) u_{t x_{i}} \nabla\left(w-\widehat{w}_{h}\right) \cdot \nabla z \\
= & I_{31}+I_{32}+I_{33} .
\end{aligned}
$$

Observing that $\|f\|_{L^{p}} \leqslant c p\|f\|_{H^{1}}$ for $p>2$ and using 2.18 , 2.21, , A.1 as well as an inverse estimate we deduce

$$
\begin{aligned}
\left|I_{31}\right| & \leqslant c\left(\left\|\nabla\left(u-\widehat{u}_{h}\right)\right\|+\left\|\nabla\left(u_{t}-\widehat{u}_{h t}\right)\right\|\right)\left(\left\|\nabla\left(I_{h} w-\widehat{w}_{h}\right)\right\|_{L^{2 p /(p-2)}}\|\nabla z\|_{L^{p}}+h\|w\|_{W^{2, \infty}}\|\nabla z\|\right) \\
& \leqslant c\left(p h h^{-2 / p}\left\|\nabla\left(I_{h} w-\widehat{w}_{h}\right)\right\|+c h^{2}\right)\|z\|_{H^{2}} \leqslant c p h^{2} h^{-2 / p}\left\|w_{t}-\widehat{w}_{h t}\right\| .
\end{aligned}
$$


The choice $p=|\log h|$ then implies that

$$
\left|I_{31}\right| \leqslant c h^{2}|\log h|\left\|w_{t}-\widehat{w}_{h t}\right\| \leqslant \epsilon\left\|w_{t}-\widehat{w}_{h t}\right\|^{2}+c_{\epsilon} h^{4}|\log h|^{2} .
$$

Clearly,

$$
\left|I_{32}\right| \leqslant c h\left\|\nabla\left(w-\widehat{w}_{h}\right)\right\|\|z\|_{H^{2}} \leqslant c h^{2}\left\|w_{t}-\widehat{w}_{h t}\right\| \leqslant \epsilon\left\|w_{t}-\widehat{w}_{h t}\right\|^{2}+c_{\epsilon} h^{4} .
$$

Integration by parts together with $\mathrm{A} .2$ yields

$$
\left|I_{33}\right|=\left|\int_{\Omega} \nabla \cdot\left(E_{p_{i}}(\nabla u) u_{t x_{i}} \nabla z\right)\left(w-\widehat{w}_{h}\right)\right| \leqslant c h^{2}|\log h|\|z\|_{H^{2}} \leqslant \epsilon\left\|w_{t}-\widehat{w}_{h t}\right\|^{2}+c_{\epsilon} h^{4}|\log h|^{2} .
$$

In order to deal with $I_{4}$ we use the splitting

$$
\begin{aligned}
I_{4}= & \int_{\Omega}\left(E_{p_{i}}\left(\nabla \widehat{u}_{h}\right) \widehat{u}_{h t x_{i}}-E_{p_{i}}(\nabla u) u_{t x_{i}}\right) \nabla w \cdot \nabla\left(I_{h} z-z\right) \\
& +\int_{\Omega}\left(E_{p_{i}}\left(\nabla \widehat{u}_{h}\right)-E_{p_{i}}(\nabla u)\right)\left(\widehat{u}_{h t x_{i}}-u_{t x_{i}}\right) \nabla w \cdot \nabla z \\
& +\int_{\Omega}\left(E_{p_{i}}\left(\nabla \widehat{u}_{h}\right)-E_{p_{i}}(\nabla u)\right) u_{t x_{i}} \nabla w \cdot \nabla z+\int_{\Omega} E_{p_{i}}(\nabla u)\left(\widehat{u}_{h t x_{i}}-u_{t x_{i}}\right) \nabla w \cdot \nabla z \\
\equiv & I_{41}+\cdots+I_{44} .
\end{aligned}
$$

Using 2.18, 2.21) and an interpolation estimate we obtain

$$
\left|I_{41}\right| \leqslant \operatorname{ch}\left(\left\|\nabla\left(u-\widehat{u}_{h}\right)\right\|+\left\|\nabla\left(u_{t}-\widehat{u}_{h t}\right)\right\|\right)\|z\|_{H^{2}} \leqslant \epsilon\left\|w_{t}-\widehat{w} h t\right\|^{2}+c_{\epsilon} h^{4} .
$$

Next, 2.19) and 2.21 imply

$$
\left|I_{42}\right| \leqslant c\left\|\nabla\left(u-\widehat{u}_{h}\right)\right\|_{L^{\infty}}\left\|\nabla\left(u_{t}-\widehat{u}_{h t}\right)\right\|\|\nabla z\| \leqslant \epsilon\left\|w_{t}-\widehat{w}_{h t}\right\|^{2}+c_{\epsilon} h^{4}|\log h|^{2} .
$$

An application of Lemma 3.1 yields

$$
\left|I_{43}\right| \leqslant c h^{2}|\log h|\|z\|_{H^{2}} \leqslant \epsilon\left\|w_{t}-\widehat{w}_{h t}\right\|^{2}+c_{\epsilon} h^{4}|\log h|^{2} .
$$

Finally, integration by parts together with 2.20 implies that

$$
\left|I_{44}\right| \leqslant c h^{2}|\log h|^{2}\|z\|_{H^{2}} \leqslant \epsilon\left\|w_{t}-\widehat{w}_{h t}\right\|^{2}+c_{\epsilon} h^{4}|\log h|^{4} .
$$

The remaining terms $I_{5}, I_{6}$ and $I_{7}$ can be dealt with in a similar manner so that we obtain A.4 after collecting the above estimates and choosing $\epsilon$ sufficiently small.

\section{Acknowledgements}

This work was supported by the Deutsche Forschungsgemeinschaft via DFG-Forschergruppe "Nonlinear partial differential equations: Theoretical and numerical analysis" and via DFGGraduiertenkolleg: "Nichtlineare Differentialgleichungen: Modellierung, Theorie, Numerik, Visualisierung". The graphical presentations were performed with the packages GRAPE and Xgraph. 


\section{REFERENCES}

1. BÄnsch, E., Morin, P., \& Nochetto, R. H. Surface diffusion of graphs: Variational formulation, error analysis and simulation. SIAM J. Numer. Anal. 42 (2004), 773-799. Zbl 1073.65098 MR 2084235

2. BURGER, M. Numerical simulation of anisotropic surface diffusion with curvature-dependent energy. J. Comput. Phys. 203 (2005), 602-625. Zbl pre02167956 MR 2122887

3. Clarenz, U., Diewald, U., Dziuk, G., Rumpf, M., \& Rusu, R. A finite element method for surface restoration with smooth boundary conditions. Computer Aided Geom. Design 21 (2004), 427-445. Zbl 1069.65546 MR 2058390

4. Deckelnick, K., \& DziUK, G. Convergence of a finite element method for non-parametric mean curvature flow. Numer. Math. 72 (1995), 197-222. Zbl 0838.65103 MR 1362260

5. DeCKelnick, K., \& DzIUK, G. Error estimates for a semi implicit fully discrete finite element scheme for the mean curvature flow of graphs. Interfaces Free Bound. 2 (2000), 341-359. Zbl 0974.65088 MR 1789171

6. Deckelnick, K., Dziuk, G., \& Elliott, C. M. Error analysis of a semidiscrete numerical scheme for diffusion in axially symmetric surfaces. SIAM J. Numer. Anal. 41 (2003), 2161-2179. Zbl 1058.65097 MR 2034610

7. Deckelnick, K., Dziuk, G., \& Elliott, C. M. Fully discrete semi-implicit second order splitting for anisotropic surface diffusion of graphs. Preprint Nr. 33/2003, Univ. Magdeburg, to appear in SIAM J. Numer. Anal.

8. Droske, M., \& Rumpf, M. A level set formulation for Willmore flow. Interfaces Free Bound. 6 (2004), 361-378. Zbl 1062.35028 MR 2095338

9. FRehSE, J., \& RANNACHER, R. Asymptotic $L^{\infty}$-error estimates for linear finite element approximations of quasilinear boundary value problems. SIAM J. Numer. Anal. 15 (1978), 418-431. Zbl 0386.65049 MR 0502037

10. Johnson, C., \& ThoméE, V. Error estimates for a finite element approximation of a minimal surface. Math. Comp. 29 (1975), 343-349. Zbl 0302.65086 MR 0400741

11. Kuwert, E., \& Schätzle, R. The Willmore flow with small initial energy. J. Differential Geom. 57 (2001), 409-441. Zbl 1035.53092 MR 1882663

12. KUWERT, E., \& SCHÄtZLE, R. Removability of point singularities of Willmore surfaces. Ann. of Math. 159 (2004), 315-357. Zbl pre02151004 MR 2119722

13. Mayer, U. F., \& Simonett, G. A numerical scheme for axisymmetric solutions of curvature-driven free boundary problems, with applications to the Willmore flow. Interfaces Free Bound. 4 (2002), 89-109. Zbl 1005.65095 MR 1877537

14. NitsChe, J. C. C. Boundary value problems for variational integrals involving surface curvatures. Quart. Appl. Math. 51 (1993), 363-387. Zbl 0785.35027 MR 1218374

15. RANNACHER, R. Some asymptotic error estimates for finite element approximation of minimal surfaces. RAIRO Anal. Numér. 11 (1977), 181-196. Zbl 0356.35034 MR 0445866

16. Rusu, R. An algorithm for the elastic flow of surfaces. Interfaces Free Bound. 7 (2005), 229-239. Zbl pre02215421 MR 2171130

17. Simonett, G. The Willmore flow near spheres. Differential Integral Equations 14 (2001), 1005-1014. Zbl pre01832854 MR 1827100

18. Willmore, T. J. Riemannian Geometry. Clarendon, Oxford (1993). Zbl 0797.53002 MR 1261641 